\title{
Genome-wide Identification and Expression Analysis of the YTH Domain-containing RNA-binding Protein Family in Citrus Sinensis
}

\author{
Zhigang Ouyang, Huihui Duan, Lanfang Mi, Wei Hu, Jianmei Chen, Xingtao Li ${ }^{1}$, and \\ Balian Zhong ${ }^{1}$ \\ National Navel Orange Engineering Research Center, College of Life Sciences, Gannan Normal \\ University, Ganzhou 341000, China
}

\begin{abstract}
AdDitional Index words. YT521-B homology, citrus, gene expression, stress response, hormone
AbSTRact. In eukaryotic systems, messenger RNA regulations, including splicing, 3 '-end formation, editing, localization, and translation, are achieved by different RNA-binding proteins and noncoding RNAs. The YTH domain is a newly identified RNA-binding domain that was identified by comparing its sequence with that of splicing factor YT521-B. Previous study showed that the YTH gene plays an important role in plant resistance to abiotic and biotic stress. In this study, 211 YTH genes were identified in 26 species that represent four major plant lineages. Phylogenetic analysis revealed that these genes could be divided into eight subgroups. All of the YTH genes contain a YT521 domain and have different structures. Ten YTH genes were identified in navel orange (Citrus sinensis). The expression profiles of these CitYTH genes were analyzed in different tissues and at different fruit developmental stages, and CitYTH genes displayed distinct expression patterns under heat, cold, salt, and drought stress. Furthermore, expression of the CitYTH genes in response to exogenous hormones was measured. Nuclear localization was also confirmed for five of the proteins encoded by these genes after transient expression in Nicotiana benthamiana cells. This study provides valuable information on the role of CitYTHs in the signaling pathways involved in environmental stress responses in Citrus.
\end{abstract}

Pre-messenger RNA (mRNA), synthesized from a DNA template in the cell nucleus by transcription, is modified by several processes to become a mature functional mRNA, which can be subsequently translated (Herzel and Neugebauer, 2015). In eukaryotic systems, pre-mRNA processing, including splicing (Braunschweig et al., 2013), 3 '-end formation, editing, and polyadenylation (Shi and Manley, 2015), is achieved via different RNA-binding proteins (RBPs) and noncoding RNAs (such as microRNAs) in the nucleus and cytoplasm. RBPs existed in association with RNA to form ribonucleoprotein complexes, and are crucial players in all aspects of mRNA regulation by binding RNA with different RNA-sequence specificities and affinities (Calabretta and Richard, 2015; Glisovic et al., 2009). RBPs are widely distributed among species. More than 200 putative RBP genes have been identified in Arabidopsis thaliana, and $\approx 250$ have been identified in rice (Oryza sativa) (Cook et al., 2010; Lorkovic, 2009). Predicted structure analyses have revealed that RBPs contain several common motifs, including RNA recognition motifs (RRMs), double-stranded RNA-binding domains (Tam et al., 2010), heterogeneous nuclear ribonucleoprotein $\mathrm{K}$ homology domains (Lorkovic and Barta, 2002), Pumilio/FBF domains (Cheong and Hall, 2006; Wang et al., 2013), Piwi Argonaute and Zwille domains (Song et al., 2003), DEAD/DEAH boxed (Owttrim, 2006), $\mathrm{ZnF}$ domains (mainly the $\mathrm{CCCH}$ type) (Kim et al., 2007;

Received for publication 22 Oct. 2018. Accepted for publication 2 Jan. 2019. This work was financially supported by grants from Key R \& D projects of Jiangxi (20171ACF60022), and Science and Technology Support Program of Jiangxi Province (20152ACF60007).

$\mathrm{BZ}$ and $\mathrm{ZO}$ designed the experiments. $\mathrm{ZO}, \mathrm{HD}, \mathrm{LM}, \mathrm{WH}$, and $\mathrm{JC}$ carried out most of the experiments. BZ, XL, and ZO drafted and revised the manuscript. All authors read and approved the final manuscript.

1CCorresponding authors. E-mail: lixt.gnnu@qq.com or bal.zh@163.com.
Qu et al., 2014), and auxiliary domains (Alba and Pagès, 1998; Ambrosone et al., 2012).

The regulatory mechanisms of RBPs, which are structurally and functionally important for various cellular processes, involve brief interactions or stable binding of target RNAs (Sharan et al., 2017). The human HuR protein is a well-studied RBP. Two RRMs within the HuR N-terminal region mediate the recognition of adenosine-uridine (AU)-rich elements located primarily within the $3^{\prime}$-untranslated region (UTR) of target mRNA transcripts. Another RRM in the C-terminus region of HuR has affinity for polyadenosine [poly(A)] RNA and is thought to bind to the poly(A) tail of target mRNAs. This binding could increase the stability of the HuR-bound target mRNA, and further increase the steady-state protein level (Wigington et al., 2014). In addition to their role in normal cellular functions, RBPs are emerging as a class of proteins involved in a wide range of posttranscriptional regulatory events that are important in providing plants with the ability to respond rapidly to changes in environmental conditions (Marondedze et al., 2016).

The YTH (YT521 homologous) domain, which is composed of 100 to 150 amino acids, is a newly identified RNA-binding domain that was identified by comparing its sequence with that of splicing factor YT521-B (Stoilov et al., 2002). Homology searches have shown that the YTH domain is typical for eukaryotes and is particularly abundant in plants (Stoilov et al., 2002; Zhang et al., 2010). The predicted secondary structure of the YTH domain consists of four $\alpha$-helices and six $\beta$-strands, and, via its conserved amino acid sequences (Trp380, Phe412, and Gly414), shows the ability to bind with singlestranded mRNA (Zhang et al., 2010). The first gene discovered to carry this domain was YT521, which was cloned from the astrocytes of rats (Rattus norvegicus), the expression of this 
gene was upregulated in reoxygenated astrocytes (Hartmann et al., 1999). Human YTH domain family proteins include five members, YTHDF1-3 and YTHDC1-2. YTHDF2 promotes mRNA degradation by binding with m6A-modified mRNA and transporting it to processing bodies (P-bodies, cytoplasmic decay sites) in the cell cytoplasm. However, the regulatory function of YTHDF1 binding is opposite that of YTHDF2. YTHDF1 enhances the translation of its targets by interacting with initiation factors (eIF3) and facilitating ribosome loading. A third member of the YTH family, YTHDF3 promotes protein synthesis synergistically with YTHDF1 and affects methylated mRNA decay via YTHDF2, suggesting that these three YTHDF proteins may act in an integrated and cooperative manner to impact fundamental biological processes related to m6A RNA methylation (Shi et al., 2017).

Proteins with a YTH domain have been reported to play roles in multiple aspects of plant life, such as developmental progress, and responses to abiotic and biotic stress. However, direct evidence is limited. Wang et al. (2017a) reported that overexpressing MhYTP1 or MhYTP2 in Malus hupehensis led to sensitivity to heat stress, high salinity, and Diplocarpon mali infection, but increased resistance to water logging, chilling stress, drought stress, and nutrition deficiency conditions. However, no biological functions of YTH domain-containing proteins have been reported in other plant species. Many plant genome projects have recently been completed, making it possible to identify YTH domain-containing proteins in different species. Recently, most studies have focused on the identification and characterization of YTH domain-containing protein family in plants. Several YTH genes have been identified in A. thaliana, rice, apple (Malus domestica), green algae (Chlamydomonas reinhardtii), moss (Physcomitrella patens), selaginella (Selaginella moellendorffi), norway spruce (Picea abies), grape (Vitis vinifera), tomato (Solanum lycopersicum), and cucumber (Cucumis sativus) (Li et al., 2014; Wang et al., 2014; Zhou et al., 2018); however, few reports of the YTH gene family in Citrus exist. In the present study, we identified and cloned $10 \mathrm{YTH}$ genes in $C$. sinensis and analyzed their expression patterns in response to the most common abiotic stresses, including salt, drought, heat, and cold stresses, as well as hormone treatments, such as abscisic acid (ABA), salicylic acid (SA), and jasmonic acid (JA). We also characterized the functions of these YTH genes in fruit development via transcriptome analyses. The results of this study will be helpful for future investigations aimed at the functional characterization of these YTH genes and their utilization for genetic improvement.

\section{Materials and Methods}

Plant materials. Citrus sinensis (L.) Osbeck cv. Gannan Zao was used for expression assays. 'Gannan Zao' navel orange seedlings were grafted and grown in plastic pots that contained a composted soil mixture (1 sand: 1 soil: 1 plant ash) under fluorescent light $\left(200 \mu \mathrm{E} \cdot \mathrm{m}^{2} \cdot \mathrm{s}^{-1}\right)$ with a $14 / 10 \mathrm{~h}$ light/dark photoperiod at 22 to $24{ }^{\circ} \mathrm{C}$. For tissue or organspecific expression of $C$. sinensis YTH genes (CitYTHs), the mature leaves, stems that had spring shoots $(\approx 2$ months after grafting to trifoliate orange plant), flowers at full bloom and whole fruits at 140, 160, and $200 \mathrm{~d}$ after flowering were collected from 4-year-old 'Gannan Zao' trees grown in a culture chamber. Lateral roots of seedlings were collected from trifoliate orange plant. Tissue materials were frozen in liquid nitrogen and stored at $-80{ }^{\circ} \mathrm{C}$ until use.

IDENTIFICATION OF YTH PROTEINS IN C. SINENSIS. BLASTP searches were performed against the National Center for Biotechnology Information (NCBI, Bethesda, MD) database and Phytozome databases (Goodstein et al., 2011) using $A$. thaliana and rice YTH proteins as queries. The protein, predicted complementary DNA (cDNA), and genomic sequences for the putative YTH genes were downloaded. Each of the YTH candidate genes was then queried against The Arabidopsis Information Resource (Lamesch et al., 2012) using the BLASTP program, and those associated with the greatest $E$ value with at least one known $A$. thaliana YTH gene were considered members of the $C$. sinensis YTH family. The Compute $\mathrm{pI} / \mathrm{Mw}$ (isoelectric point/molecular weight) tool of ExPASy (Gasteiger et al., 2005) was used to calculate the Mw and $\mathrm{pI}$ of the deduced $C$. sinensis $\mathrm{YTH}$ proteins. The queries for the conserved domains were performed using the NCBI Conserved Domain Database [NCBI-CDD (Marchler-Bauer et al., 2014)], and the gene structures were analyzed with the online Gene Structure Display Server [GSDS (Li et al., 2014)].

Multiple Sequence alignment and Phylogenetic ANALYSIS. Protein sequences of other plants, including $A$. thaliana, rice, tomato, and rapeseed were obtained from the NCBI database, the Vitis vinifera Genome Database [VvGDB (Duvick et al., 2008)] and the Phytozome database. Multiple sequence alignments of the full-length YTH proteins from representative plant species were performed using the ClustalX program (Larkin et al., 2007), and were viewed by GeneDoc (Nicholas et al. 1997). A phylogenetic tree was constructed using MEGA 6.0 software via the neighborjoining (NJ) method with 1000 bootstrap replicates (Tamura et al., 2013).

Abiotic STRess AND hormone Treatments. After they were grafted, three pots of 3-week-old navel orange seedlings (three biological replicates) with a consistent growth status for each group were chosen and treated with abiotic stresses and hormone treatments. With respect to drought and salinity stress, the seedlings were transferred from hydroponic cultivation to a layered filter for fast dehydration or maintained on liquid media supplemented with $200 \mathrm{~mm} \mathrm{NaCl}$ for salinity stress. With respect to cold and heat treatments, culture tubes containing navel orange seedlings were placed in incubators that were maintained at $4 \pm 1$ or $42 \pm 1{ }^{\circ} \mathrm{C}$, respectively. The navel orange seedlings maintained at $28 \pm 1{ }^{\circ} \mathrm{C}$ were used as negative controls. With respect to hormone treatments, 3-week-old navel orange seedlings with expanded leaves were sprayed with $100 \mathrm{~mm}$ SA (Sigma-Aldrich, Poole, UK), $100 \mu \mathrm{M}$ methyl jasmonate [MeJA (Sigma-Aldrich)], or $100 \mu \mathrm{M}$ ABA (SigmaAldrich) in solutions containing $0.1 \%$ ethanol and $0.02 \%$ polyoxyethylene sorbitol anhydride monolaurate (Tween-20; Sigma-Aldrich) and then maintained under normal growth conditions. Plants sprayed with solutions that contained only $0.1 \%$ ethanol and $0.02 \%$ Tween- 20 were used as negative controls. All leaf samples harvested at the indicated times were stored at $-80{ }^{\circ} \mathrm{C}$ until future use.

RNA EXTRACTION AND GENE EXPRESSION ANALYSES. Total RNA was isolated from plants using TRIZOL reagent (Invitrogen, Shanghai, China) and subsequently reverse transcribed into cDNA using Superscript III RT (Invitrogen) in accordance with the manufacturer's protocols. Fluorescence quantitative polymerase chain reaction (qPCR) was used to 
investigate the expression patterns of CitYTHs. The qPCR was conducted in a total volume of $25 \mu \mathrm{L}$, which consisted of 1-mg aliquots of cDNA, $12.5 \mu \mathrm{L}$ of SYBR Green Master Mix Reagent (TaKaRa, Kyoto, Japan), and specific primers (3 pmol). qPCR was performed on a 96-well plate qPCR system (CFX96; Bio-Rad, Hercules, CA) in accordance with the following profile: $5 \mathrm{~min}$ at $94^{\circ} \mathrm{C}$ followed by 30 cycles of $30 \mathrm{~s}$ at $94{ }^{\circ} \mathrm{C}, 30 \mathrm{~s}$ at $57^{\circ} \mathrm{C}$, and $1 \mathrm{~min}$ at $72^{\circ} \mathrm{C}$. Quantification analysis was carried out via CFX Manager Software (Bio-Rad, Hercules, CA). The qPCR primers used are listed in Supplemental Table 1, and $C$. sinensis CitActin (accession no. XM_006464503) was used as an internal control in conjunction with primers CitActin-F and CitActin-R (Lugassi et al., 2015). Each experiment was performed in at least three technical replications and three biological replicates were run per sample (using three independent sets of RNA).

Subcellular localization. The coding sequences of the CitYTH genes were amplified by PCR with specific primers (Supplemental Table 1). The PCR products were then digested with $X b a \mathrm{I}$ and SamI restriction enzymes and subsequently inserted into the appropriate sites in pFGC-Egfp vectors to obtain pFGC-Egfp:CitYTH constructs. The sequence-verified constructs and pFGC-Egfp vectors were transformed into Agrobacterium tumefaciens GV3101. Four-week-old $N$. benthamiana plants were inoculated with the transformed A. tumefaciens cultures and then grown at $25{ }^{\circ} \mathrm{C}$ for $36 \mathrm{~h}$. Fluorescence signals were excited at $488 \mathrm{~nm}$ and detected using a 500- to 530-nm emission filter, both of which were performed with a confocal laser scanning microscope (LSM 510 Meta confocal microscope; Zeiss, Oberkochen, Germany).

RNA SEQuencing. Transcriptome sequencing (RNA-Seq) analysis was used to study the expression of CitYTH genes during different fruit development stages. Transcriptome sequencing and assembly were performed by Meiji Biological Medicine Technology Co., Ltd. (Shanghai, China). A heatmap for the expression of CitYTH genes was generated in R using the heatmap 2 function from the gplots CRAN library (Warnes et al., 2013).

Statistical analysis. SAS version 9.4 (SAS Institute, Cary, NC) was used for the statistical analyses. The experiments were repeated three times, and three replicates were included per experiment. The data from three independent experiments were analyzed via Dunnett's $t$ test, taking $P<0.05$ as a significant difference.

\section{Results}

IDENTIFICATION OF YTH DOMAIN-CONTAINING FAMILY MEMBERS IN 26 PLANT SPECIES. Based on domain composition analyses of sequences retrieved from BLAST searches using $A$. thaliana YTH domain-containing proteins as queries, $211 \mathrm{YTH}$ sequences were identified among 26 plant species, including six Chlorophyta, one moss, one Lycopodiatae, four monocot, and 15 eudicot species (Fig. 1). Detailed information on these YTH family genes is provided in Supplemental Table 2. With the exception of that of the reported YTH gene, the identified sequences were named in accordance with their identity with AtYTH1. The evolutionary history of the plants suggested that the first plant YTH gene occurred in Chlorophyta, but only half of the Chlorophyta species contained the YTH gene. The presence of YTH domain-containing proteins varied in all other tested plant species (Fig. 1), suggesting that the YHT domain-containing family is universally present in multicellular land plants but not in unicellular eukaryotes. In addition, the size of the YHT family differed among the moss, lycophyte, and flowering higher plant species. The lycophyte $S$. moellendorffii and the moss $P$. patens have only two and four YHT genes, respectively, whereas excluding grand eucalyptus (Eucalyptus grandis), potato (Solanum tuberosum), and cucumber, flowering higher plant species generally carry 8 to 20 YTHs. Many plants were found to have more than 10 YTH genes (Fig. 1, Supplemental Table 2).

Phylogenetic analyses of YTH Genes. To investigate the phylogenetic relationships among YTH proteins, a phylogenetic tree of YTH proteins from 26 plant species was constructed based on the alignment of full-length proteins by the NJ method. The phylogenetic analyses revealed a clear clustering of the 211 YTH family members into three groups, I, II, and III; group I was further divided into two subgroups, whereas both group II and III were separated into three subgroups (Fig. 2). With respect to the eudicot plants, all YTH proteins were distributed in group Ia, IIc, IIIa, IIIb, and IIIc, except for $E$. grandis, $S$. tuberosum, and $C$. sativus. Similarly, apart from those of maize (Zea mays), the YTH proteins of grass species were clustered in group Ia, IIb, IIc, IIIa, IIIb, and IIIc. Z. mays has the second largest YTH family, and its members distributed in almost all subgroups excluding group Ia. Two species, $S$. moellendorffii and $P$. patens, have fewer YTH members, all of which are distributed in group Ia and IIIb; these members share high identity among their protein sequences. Among six select Chlorophyta species, only three were found to contain YTH proteins (one YTH each), and they were all clustered in the group Ib. Interestingly, one ZmYTH (ZmYTH18) was also congregated in this group.

Gene STRUCTURE ANALYSIS OF THE YTH-CONTAINING FAMILY MEMBERS. To determine the possible gene structure relationships among YTH gene orthologs and paralogs, the exon/intron structures of individual YTH genes identified from 26 plant species were analyzed using GSDS software. The number of introns in these genes varied widely from 2 to 13 (Fig. 3). Two groups (Ib and IIb) with relatively few YTH gene members also contained few introns. Group Ib members had 3 or 4 introns, and group IIb members had 5 or 6 introns. Group Ia YTH genes contained introns whose numbers ranged from 4 (AtYTH1) to 9 (PpYTH4), although most had $6(53 \%)$ or 7 (21\%), and group IIc YTH genes had 6 (5 genes) to 10 ( $V v Y T H 9)$ introns, although most had $8(28 \%)$. Group IIIa YTH genes had 4 (CitYTH5) to 9 (VvYTH4 and PpYTH4) introns, while most had $8(57 \%)$ or $6(32 \%)$, and group IIIb YTH genes had 2 (PvYTH2) to 13 (PpYTH2) introns, although most had $6(28 \%)$. The structure of the YTH genes in group IIIc distinctly differed from that in the other groups, with more introns at different phases and with different lengths. The number of introns in group IIIc ranged from 2 (ZmYTH6) to 9 ( $V v Y T H 8)$; most members had $7(55 \%)$. Moreover, the intron phases within the YTH gene families varied greatly among the different groups. For instance, most group Ia YTH genes had the unique phase pattern 2-2-0-0-0-0 (45\%) for 6-intron members and 1-0-0$2-0-0-2(18 \%)$ for 7 -intron members, whereas the pattern 1 0-0-0-0-0 for 6-intron members and 1-0-1-0-0-0 (48\%) for 7 -intron members were observed in group IIIa and group IIIc genes, respectively. Notably, the phase patterns of different introns within the same group were very similar. For instance, the phase patterns for 7-intron, 8-intron, and 9-intron members were 1-0-1-0-0-0-0, 1-0-1-0-0-0-0-1 and 


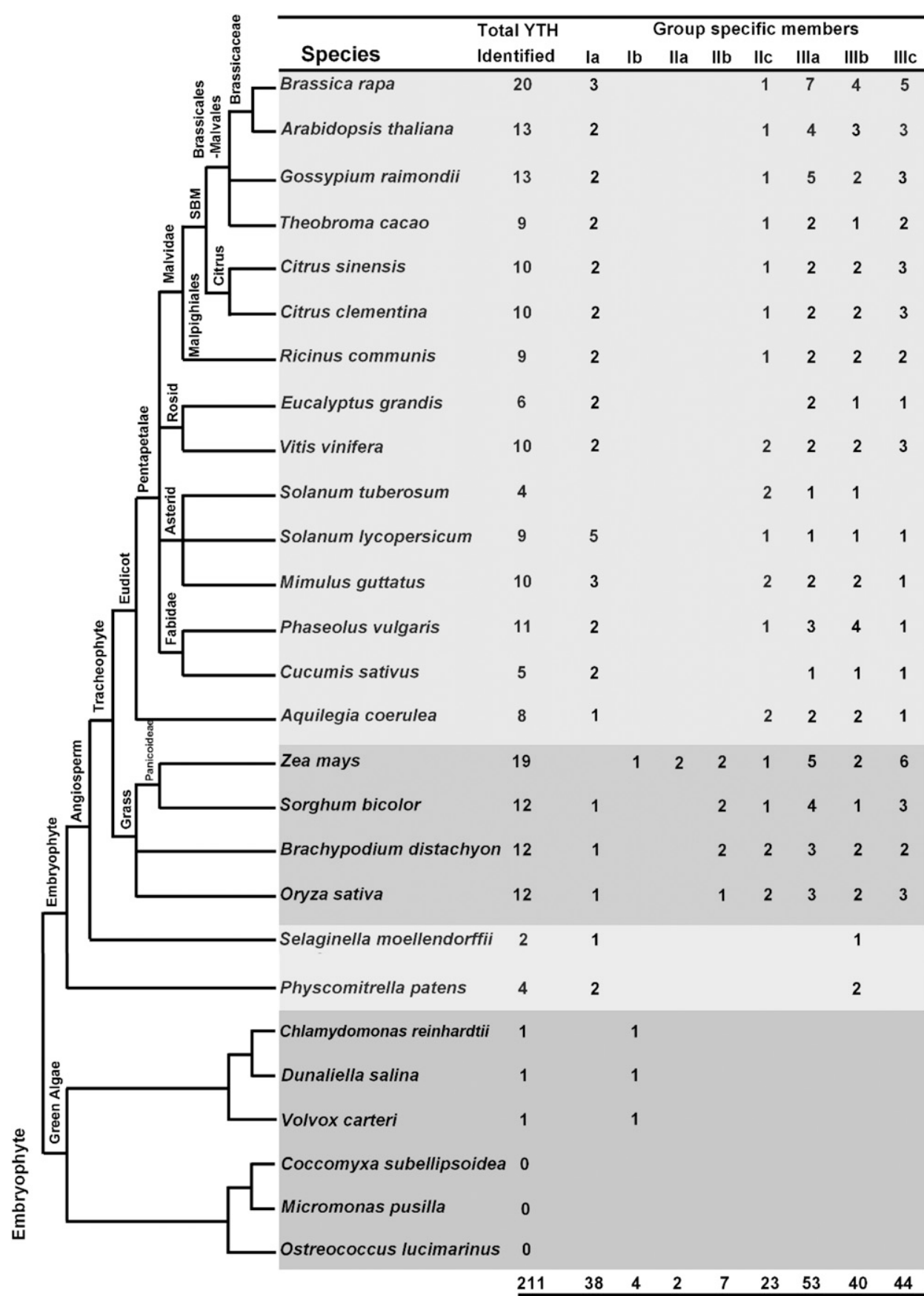

Fig. 1. Species of plant investigated in this study. Left column: phylogenetic tree of plant species generated according to the classification of plants in the Phytozome database (Goodstein et al., 2011). Right column: YTH domaincontaining proteins identified. A total of $211 \mathrm{YTH}$ sequences were identified among 26 plant species.

$1-0-1-0-1-0-0-0-1$ in group IIIa, respectively, whereas the patterns were $1-0-0-0-0-1$ and $2-1-0-0-0-1$ for 5 -intron members and 6-intron members in group Ib, respectively.

All of the identified YTH proteins contain a YT521-B-like domain (pfam04146) (Fig. 3). In addition, ZmYTH16 possesses a prephenate dehydratase domain (PDT PF00800) (Fig. 3). Prephenate dehydratase plays function in amino acid transport and metabolism (Pascual et al., 2016). Moreover, a UBA/TS-N domain (PF00627) was found in PpYTH1 and PpYTH2 protein (Fig. 4). Ubiquitin-mediated proteolysis is involved in the regulated turnover of proteins required for controlling cell cycle progression (Liu et al., 2006). These results suggested that the YHT proteins may have other functions in addition to binding with RNA.

Tissue-sPeCIFIC EXPRESSION PATterns OF CitYTH genes. To determine the possible physiological roles of CitYTH genes (listed in Supplemental Table 2) in growth and development, we analyzed their tissue-specific expression patterns in five different tissues (roots, stems, leaves, flowers, and fruits) by qPCR. The results revealed different expression patterns of the CitYTH genes in the five tissues. As shown in Fig. 4, 10 CitYTH genes were constitutively expressed in all tested tissues but exhibited different expression patterns. Both CitYHT1 and CitYHT10 were expressed specifically in the leaves, but in the roots, stems, flowers, and fruits, their expression levels were very low. On the other hand, CitYTH5 and CitYTH9 were highly expressed in the stems, leaves, flowers, and fruits, but their expression levels in the roots were low. CitYHT3 and CitYHT6 were highly expressed in the stems and leaves, but their expression levels in the roots, flowers, and fruits were low. In addition, the expression of CitYHT4 was relatively high in the leaves, flowers, and fruits but low in the roots and stems. In general, with the exception of those of CitYTH10, the expression levels of the CitYTH genes were lowest in the roots, whereas the levels of all 10 CitYTH genes, 5 CitYTH genes (CitYTH2, 3, 4, 5, 9), and 3 CitYTH genes $(\mathrm{CitYTH2}, 5,9)$ were relatively high in the leaves, flowers, and fruits, respectively.

EXPRESSION OF CITYTH GENES IN F R U I T S A T D I F F E R E N T DEVELOPMENTAL STAGES. To study the potential role of CitYTH genes in fruit development, we evaluated their expression patterns during three different developmental stages in two different cultivars of navel orange. The time to maturity of 'Gannan Zao' is shorter than that of 'Newhall' navel orange. The results showed that the expression in two different parts of the fruits (peel and pulp) exhibited different patterns (Fig. 5). In both the 'Newhall' and 'Gannan Zao', CitYTH3, 4 and 6 were relatively highly expressed in peels, but their expression levels were low in the pulp during all three developmental stages. On the other hand, the expression levels of CitYTH8 and CitYTH9 were relatively high in the navel orange peels but low in the pulp 


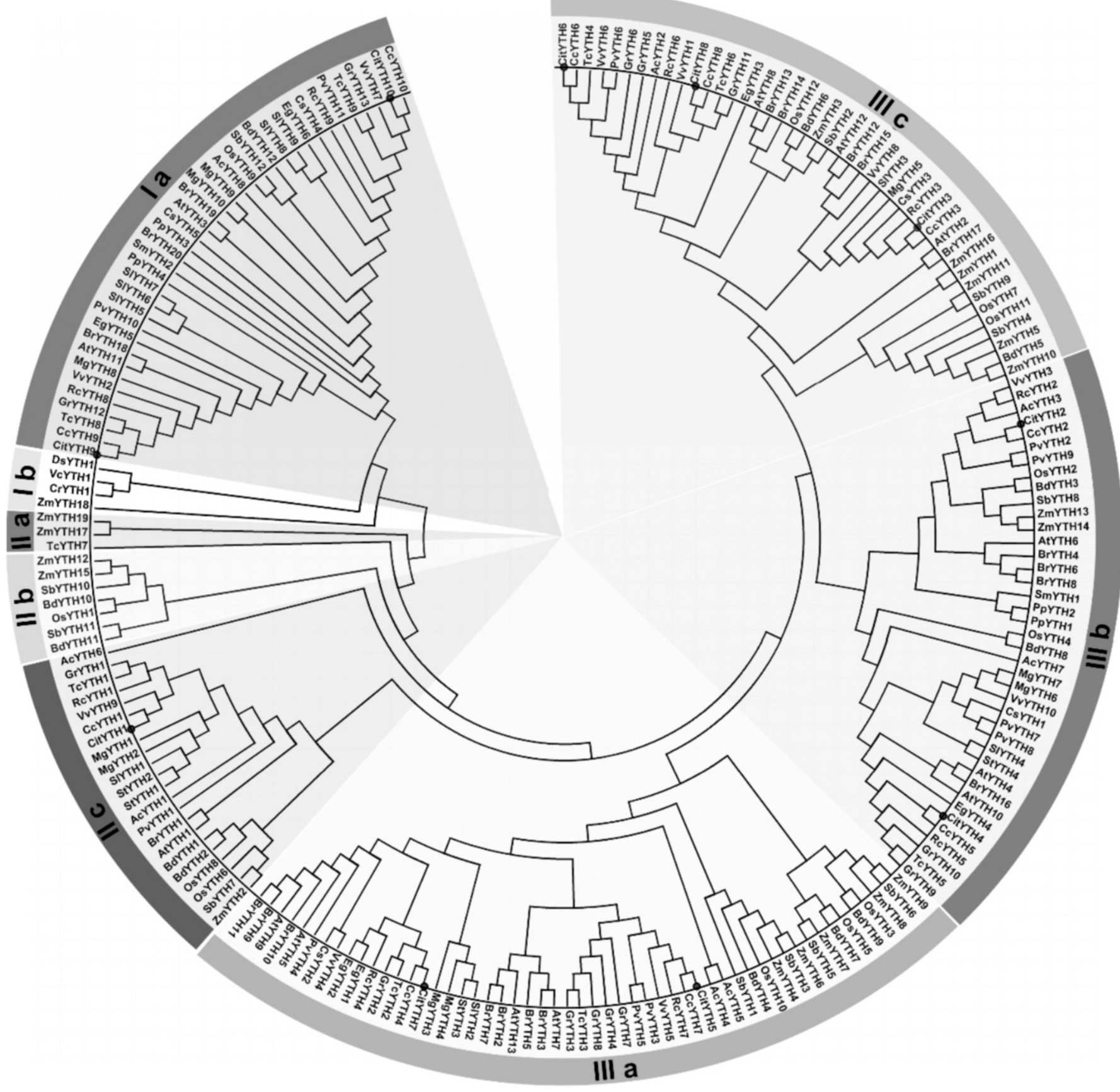

Fig. 2. Phylogenetic relationship between YTH domain-containing proteins (YTHs) identified from 26 plant species. YTH family members were clustered into three groups, and further divided into two, three, and three subgroups, respectively. Protein sequences of YTH domain-containing proteins were obtained from the Phytozome database (Goodstein et al., 2011). Multiple sequence alignments of the full-length YTH proteins from representative plant species were performed using the ClustalX program (Larkin et al., 2007). The phylogenetic tree was constructed using MEGA 6.0 by the neighbor-joining method (Tamura et al., 2013). Citrus sinensis YTH proteins are highlighted by the black circle.

from 160 to $180 \mathrm{~d}$ after full bloom (DAFB). In addition, the expression level of CitYTH5 in the navel orange peels was highest at 200 DAFB compared with other periods. In the peels, the expression of CitYTH10 in 'Gannan Zao' was higher than that in the 'Newhall', whereas the expression of CitYTH7 was lower in 'Gannan Zao' than in 'Newhall' from 160 to 200 DAFB. Furthermore, the expression of CitYTH1 and CitYTH9 was higher at 200 DAFB in 'Newhall' than in 'Gannan Zao'. As in the pulp, increased expression levels of CitYTH1, CitYTH2, and CitYTH7 from 160 to 200 DAFB in the 'Newhall' were observed. However, the expression levels of CitYTH3 and CitYTH8 were slightly higher in the 'Gannan Zao' than in the 'Newhall' at 200 DAFB, but the expression of CitYTH9 was lower. Furthermore, there were no significant differences between the expression of other genes from 160 to 200 DAFB in the pulp of the two different cultivars.

EXPRESSION PATTERNS OF YTH GENES IN RESPONSE TO ABIOTIC STRESS. Drought, high salinity, and extreme temperature are the 

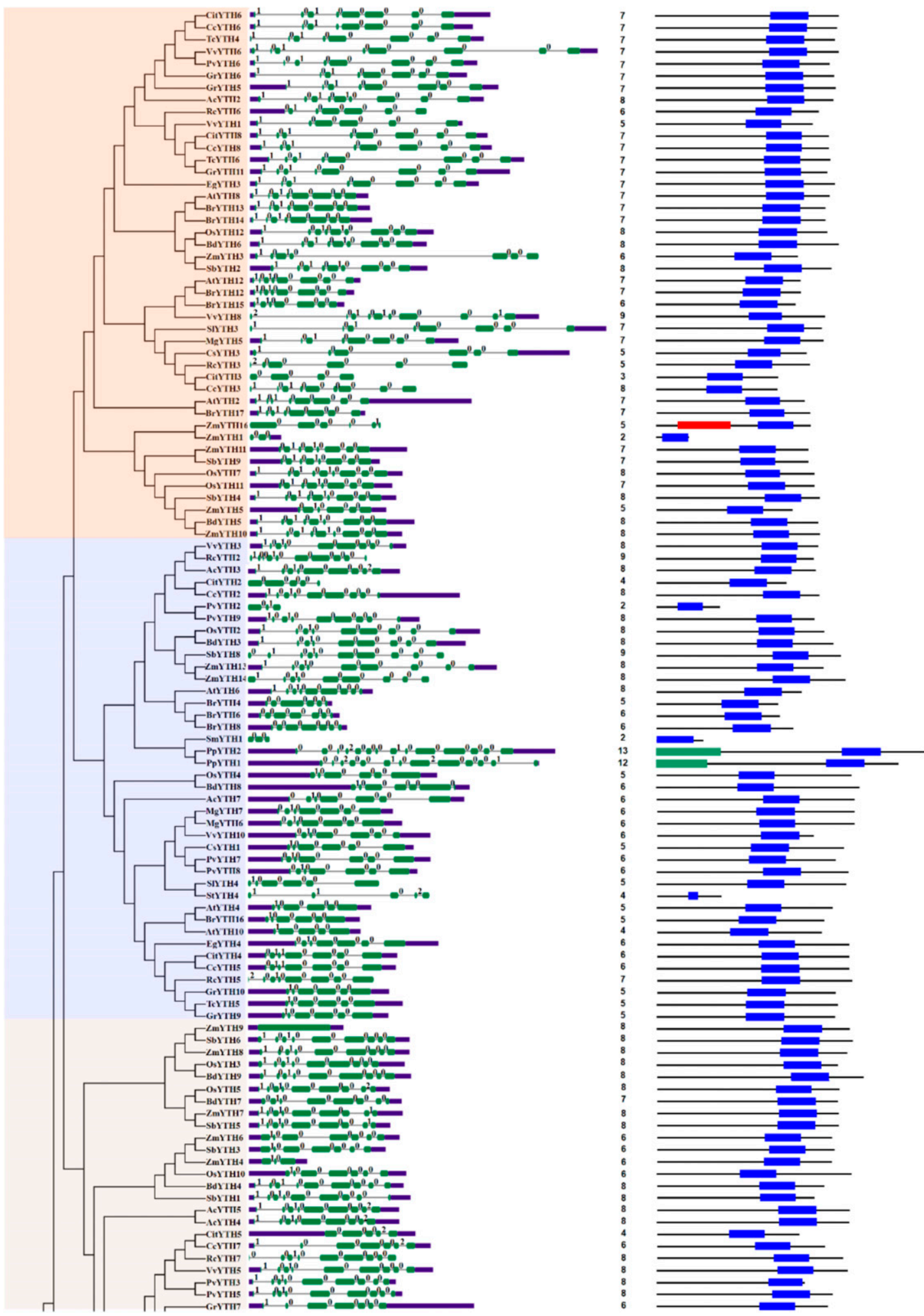

Fig. 3. Phylogenetic relationships, conserved motif compositions, numbers of introns, and gene structure of YTH domain-containing proteins. Left column: phylogenetic tree of YTH domain-containing proteins. Middle left column: exon/intron gene structure (filled box $=$ exon, solid line $=$ intron). The intron phases are indicated as the numbers 0, 1, and 2. Excluding DsYTH1, PvYTH11, SlYTH7, SlYTH8, SlYTH9, VvYTH2, VvYTH7, VvYTH9, and ZmYTH19, whose long introns are denoted by a "/", the exons and introns are drawn to scale. Middle right column: numbers of introns. Right column: conserved domains within the YTH domaincontaining proteins. The domains and motifs are drawn to scale.

major environmental stresses that affect plant growth and development and ultimately crop yields (Shahbaz and Ashraf, 2013; Zhu, 2016). To investigate the potential involvement of CitYTH genes in abiotic stress responses, the expression patterns of CitYTHs in navel orange in response to salt, drought, cold, and heat stresses were analyzed.

We first analyzed the expression of the CitYTH genes in the leaves of navel orange seedlings after they were treated with 

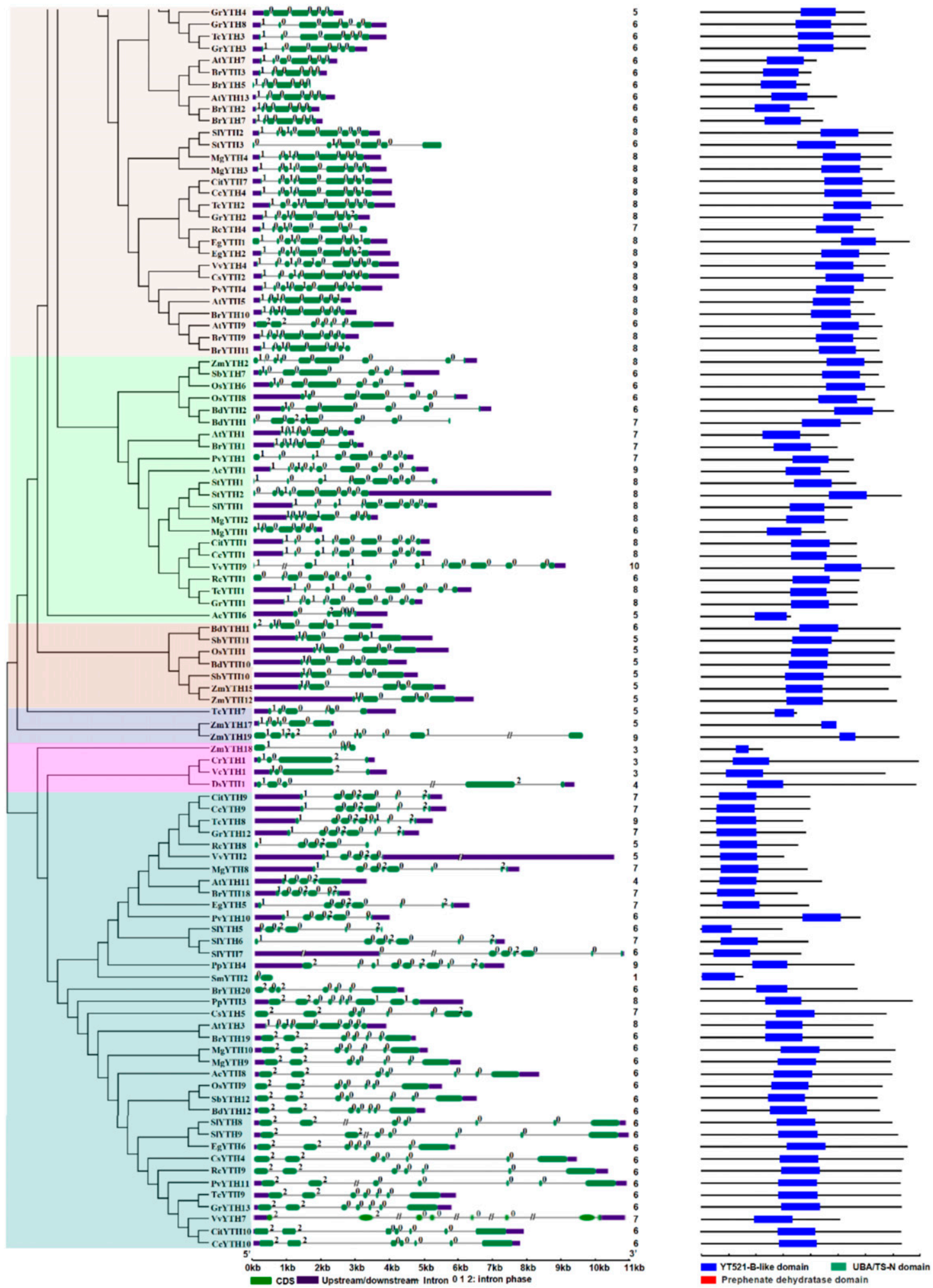

Fig. 3. (Continued)

salt $(\mathrm{NaCl})$ and drought. In our study, $10 \mathrm{CitYTH}$ genes exhibited different expression patterns under salt $(\mathrm{NaCl})$ and drought stress (Fig. 6). Compared with those in the control plants, the expression levels of CitYTH2, CitYTH3, CitYTH5, CitYTH7, and CitYTH8 were dramatically upregulated by different amounts and peaked at 24 to $48 \mathrm{~h}$ after the drought treatment. The expression of CitYTH9 peaked at $24 \mathrm{~h}$ after drought stress treatment but then decreased afterward. In addition, the expression of CitYTH1 and CitYTH4 was strongly repressed in response to drought stress, especially that of CitYTH1, whose expression decreased by $\approx 10$-fold at $48 \mathrm{~h}$ after treatment. In the salt 


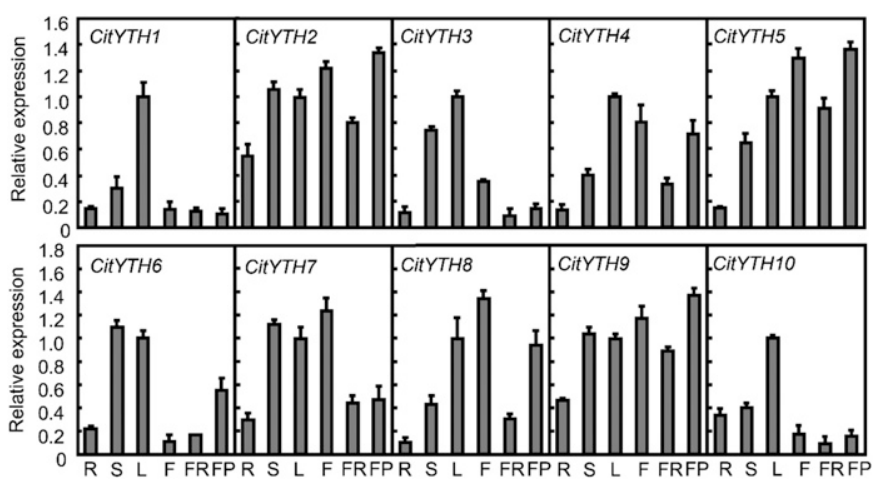

Fig. 4. Expression analysis of Citrus sinensis YTH domain-containing protein genes in the different tissues of 'Gannan Zao' navel orange plants ( $\mathrm{R}=$ roots, $\mathrm{S}=$ stems, $\mathrm{L}=$ leaves, $\mathrm{F}=$ flowers, $\mathrm{FR}=$ mature fruit pulp, $\mathrm{FP}=$ mature fruit peels). Root, stem, and leaf samples were collected from spring shoots $(\approx 2$ months after grafting). Flowers at full bloom and whole fruits at $140 \mathrm{~d}$ after full bloom were collected from adult navel orange trees grown in a culture chamber. The transcript levels of CitYTH genes were detected using the realtime polymerase chain reaction. The data presented are mean \pm SD of three independent experiments. The $\mathrm{x}$ - and $\mathrm{y}$-axes represent the different tissues of navel orange and relative expression (fold change relative to actin transcript values), respectively.

treatment assay, only increased expression of CitYTH4 was observed. In contrast, the expression of CitYTH2 was downregulated during the 24- to 48-h test period. Moreover, the expression of CitYTH6 and CitYTH8 increased at $24 \mathrm{~h}$ but then decreased at $48 \mathrm{~h}$ after treatment. The expression of CitYTH5 increased but then decreased at 24 and $48 \mathrm{~h}$ after treatment, respectively. The expression of the other five CitYTH genes (CitYTH1, 3, 7, 9) exhibited no detectable changes within $48 \mathrm{~h}$ period.

As shown in Fig. 7, the expression of four CitYTH (CitYTH2, 4, 5, 9) genes was significantly induced and peaked at $24 \mathrm{~h}$ after heat treatment; compared with that in the control plants, the expression in the treated plants increased 2.3- to 8.0-fold. However, the expression of CitYTH3, CitYTH6, CitYTH7, and CitYTH10 was downregulated at 24 or $48 \mathrm{~h}$ after heat treatment. Among the expression of these genes, the expression of CitYTH6 was repressed gradually and reached a minimum of a decrease of 10 -fold at $48 \mathrm{~h}$ after heat treatment. The expression of the other two CitYTH genes (CitYTH1 and CitYTH8) was not markedly affected under heat stress conditions. In the cold stress treatment group, the expression levels of all 10 CitYTH genes were higher (to varying degrees) than those in the control plant group under low temperature $\left(4^{\circ} \mathrm{C}\right)$ conditions. However, the patterns among them were not the same. Compared with those in the control plants, the expression levels of seven CitYTH (CitYTH1, 3, 5, 7, 8, $9,10)$ genes in the treated plants increased 1.8- to 3.3-fold and peaked at $24 \mathrm{~h}$ after the cold treatment. Furthermore, the expression levels of the other three CitYTH genes increased 3.5- to 7.2-fold and peaked at $24 \mathrm{~h}$ after cold treatment.

EXPRESSION OF CITYTH GENES IN RESPONSE TO HORMONE TREATMENTS. To examine the possible effects of SA, JA, and ABA on CitYTH transcript levels, we analyzed the expression of these genes in response to spraying with JA, SA, and ABA. As shown in Fig. 8, the expression patterns of

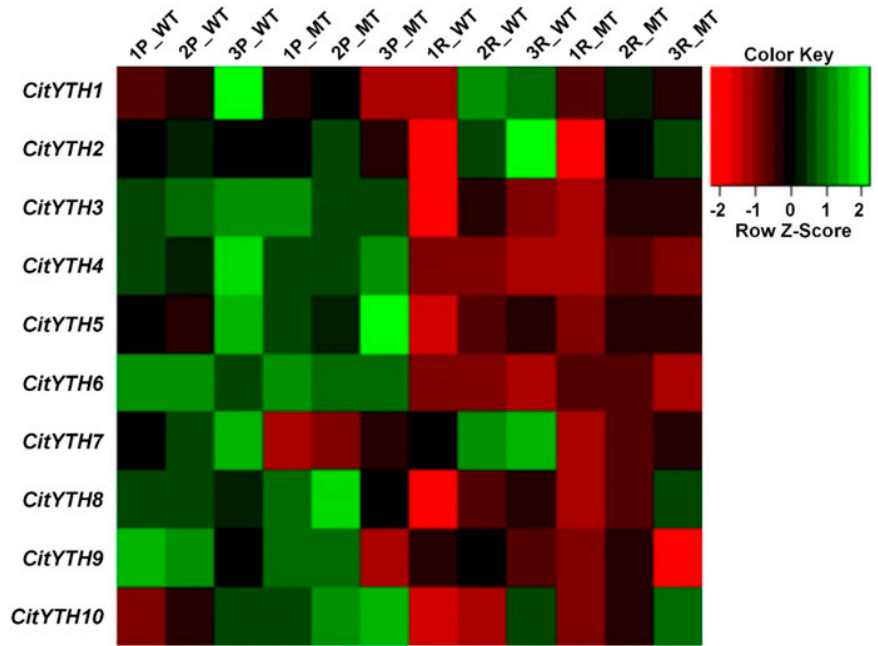

Fig. 5. Heatmap representation of Citrus sinensis YTH domain-containing protein genes in fruit peels and pulp during fruit development. Whole fruits at 140, 160, and $200 \mathrm{~d}$ after full bloom (DAFB) were collected from 4-year-old 'Gannan Zao' navel orange trees. Transcriptome sequencing (RNA-Seq) analysis was used to analyze the expression of CitYTH genes, and each experiment contains three biological replications. The heatmap was generated in R using the heatmap 2 function from the gplots CRAN library (Warnes et al., 2013). The bar on the right of heatmap represents the relative expression value. 1P_WT, 2P_WT, and 3P_WT = peels of 'Newhall' fruit at 160, 180, and 200 DAFB, respectively; 1P_MT, 2P_MT, and 3P_MT = peels of 'Gannan Zao' fruit at 160, 180, and 200 DAFB, respectively; 1R_WT, 2R_WT, and 3R_WT $=$ pulp of 'Newhall' fruit at 160, 180, and $200 \mathrm{DAFB}$, respectively; 1R_WT, 2R_WT, and 3R_WT = pulp of 'Gannan Zao' fruit at 160,180 , and $200 \mathrm{DAFB}$, respectively.

CitYTH3, CitYTH5, CitYTH6, CitYTH7, CitYTH8, and CitYTH9 were similar from 24 to $48 \mathrm{~h}$ and displayed no detectable changes after applications of SA, JA, and ABA, compared with those in the control plants. Increased expression of CitYTH2 and CitYTH10 was observed after applications of JA and SA, whereas the expression of CitYTH1 was downregulated. In response to ABA, the expression levels of both CitYTH2 and CitYTH4 were downregulated after 24 and $48 \mathrm{~h}$.

C. SINENSIS YTH DOMAIN-CONTAINING PROTEINS TARGET TO NUCLEUS AND CYTOPLASM. To investigate the subcellular localization of CitYTH proteins, the coding sequences of the CitYTH genes were amplified and fused to the N-terminus of green fluorescent protein (GFP) under the control of the CaMV 35S promoter, after which the constructs were transiently expressed in $N$. benthamiana leaves via agroinfiltration. The subcellular localization of GFP in N. benthamiana leaves was subsequently examined by confocal microscopy. The results showed that both the CitYTH:GFP and GFP alone were localized to in the nucleus and the cytoplasm (Fig. 9). These results demonstrate that CitYTH proteins do not target specific compartments and are likely to distribute ubiquitously in cells.

\section{Discussion}

YT521-B is present only in vertebrate genomes (Zhang et al., 2010). However, genome-wide identification of gene family members containing YT521-B-like domain has been reported in several plant species (Li et al., 2014; Wang et al., 


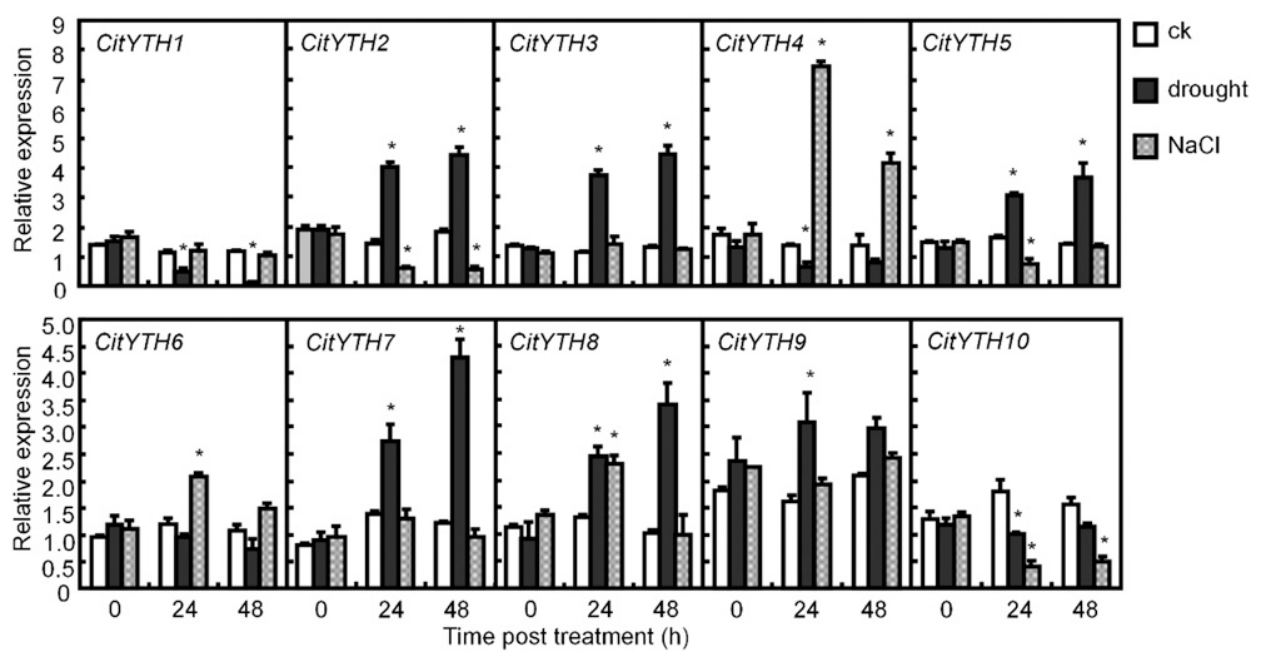

Fig. 6. Expression analysis of Citrus sinensis YTH domain-containing protein genes in 'Gannan Zao' navel orange leaves treated with drought or salt stress. Drought stress was applied to 3-week-old navel orange seedlings after grafting by transferred to three layers of filter papers for fast dehydration. Salt stress was applied to 3-week-old seedlings by drenching the plants with a $200-\mathrm{mm} \mathrm{NaCl}$ solution. Navel orange leaves were collected for quantitative polymerase chain reaction analysis at 0,24 , and $48 \mathrm{~h}$ after treatment. Relative expression folds were calculated after normalization with CitActin transcript values. The $\mathrm{x}$ - and y-axes represent the treatment time (units $=$ hours) and relative expression, respectively. The data presented are mean \pm SD of three independent experiments run in triplicate. The * above the columns indicates significant differences at the $P<0.05$ level.

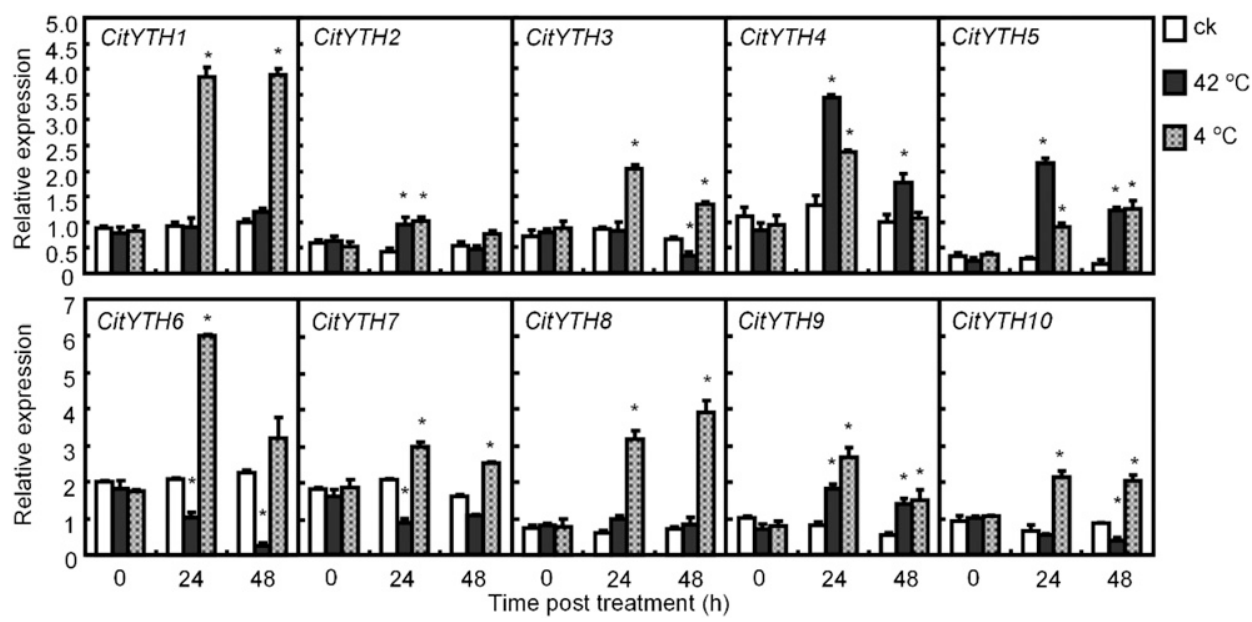

Fig. 7. Expression analysis of Citrus sinensis YTH domain-containing protein genes in 'Gannan Zao' navel orange leaves treated with cold or heat stress. Cold and heat stress was applied by placing 3-week-old navel orange seedlings after grafting in growth chambers whose temperatures were set at 4 and $42{ }^{\circ} \mathrm{C}$, respectively; navel orange trees grown at $28^{\circ} \mathrm{C}$ were used as controls. Leaf samples for quantitative polymerase chain reaction analysis were collected at 0,24 , and $48 \mathrm{~h}$ after treatment. The $\mathrm{x}$ - and $\mathrm{y}$-axes represent the treat time (units = hours) and relative expression (fold change relative to actin transcript values), respectively. The data presented are mean \pm SD of three independent experiments run in triplicate. The * above the columns indicates significant differences at the $P<0.05$ level.

2014; Zhang et al., 2010). Our sequence search in which $A$. thaliana and O. sativa $\mathrm{YTH}$ proteins were used as queries for YTH domain-containing genes from unicellular green algae, mosses, lycophytes, monocots, and eudicots suggested that YTH genes existed not only in land plants, but also in an aquatic plant (Fig. 1). Among the plant species we studied, the most ancestral to present a YTH domain-containing gene was a green alga. However, not all green algae contained the YTH gene; three green alga species (Coccomyxa subellipsoidea, Micromonas pusilla, Ostreococcus lucimarinus) lacked the YTH gene, and another three green alga species had only one YTH gene, respectively. In contrast, all other higher plant possesses YTH genes.

A combined $\mathrm{NJ}$ tree was also constructed to investigate the phylogenetic relationships of YTH genes in plants and their evolutionary relationships. The YTH members were divided into three groups, and group $\mathrm{a}, \mathrm{b}$, and $\mathrm{c}$ contained two, three, and three subgroups, respectively. The phylogenetic tree suggested that a close relationship exists among YTH genes in plant species (Fig. 2). All of the YTHs in green algae were clustered in subgroup Ib. It appeared that subgroup Ib members (apart from ZmYTH) were present in the lower plant species. The lycophyte $S$. moellendorffii and moss $P$. patens had only two and four YHT genes, respectively, and those genes were clustered into group Ia and group IIIb, whereas flowering higher plant species generally harbored 8 to $20 \mathrm{YTH}$ genes. Interestingly, the proportion of total YTH genes between $S$. moellendorffii and $P$. patens was $1: 2$, which is consistent with the proportion of the YTH genes in the corresponding subgroups. Excluding four species (S. tuberosum, E. grandis, C. sativus, and Z. mays), most angiosperm plants had group Ia, IIc, IIIa, IIIb, and IIIc YTH genes. We found $10 \mathrm{YTH}$ genes in C. sinensis, which is a smaller number than that in A. thaliana (13 members), O. sativa (12 members), $Z$. mays (19 members), and cabbage (Brassica rapa) (20 members), suggesting that the number of YTH genes is not proportional to genome size.

Gene fusion is a well-known process in molecular evolution (Enright and Ouzounis, 2001). We analyzed the gene structure of YTH. The YTH genes that clustered in the same group had similar motif distributions and exon/intron organizations (Fig. 3), implying that the YTH gene family in different species may evolve independently. For example, the phase patterns of 7-intron members, 8 -intron members, and 9intron members are 1-0-1-0-0-0-0, 1-0-1-0-0-0-0-1, and 1-01-0-1-0-0-0-1 in group IIIa, respectively, whereas the patterns for 5-intron members and 6-intron members are 1-0-0-0-1 and 2-1-0-0-0-1 in group $\mathrm{Ib}$, respectively. Similar to all of the YTH genes characterized in various species, all eight family members contain conserved YT521-B-like domains (Fig. 3). However, other motifs were also found within the YTH 


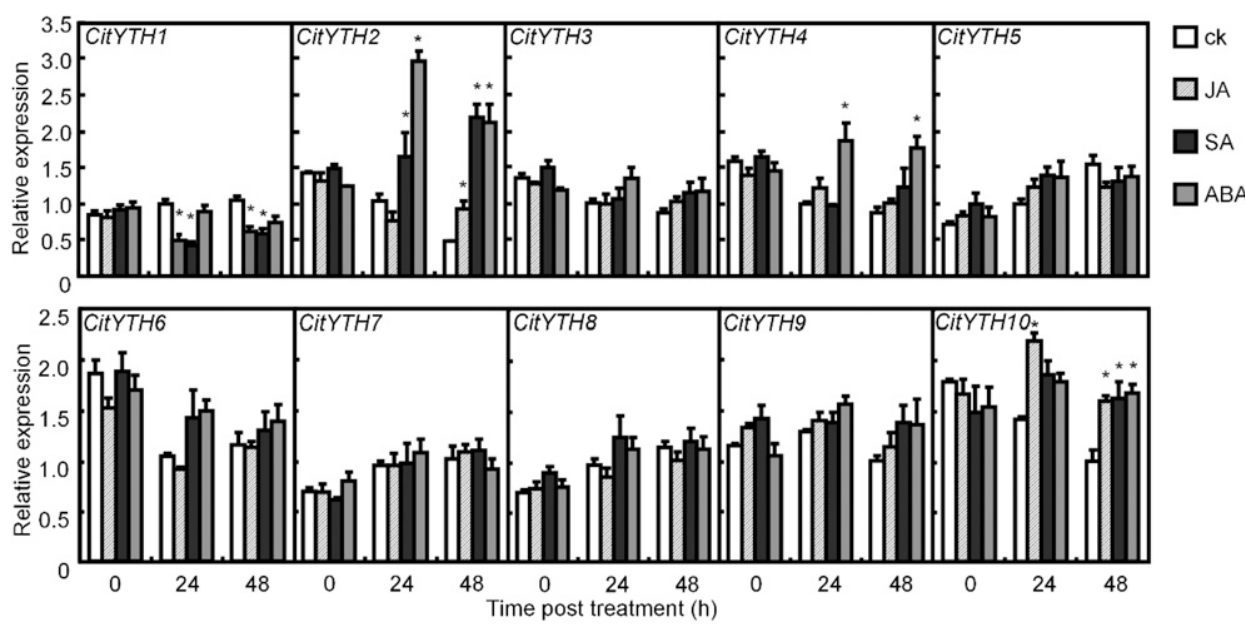

Fig. 8. Expression analysis of Citrus sinensis YTH domain-containing protein genes in 'Gannan Zao' navel orange leaves treated with salicylic acid (SA), methyl jasmonate (JA), and abscisic acid (ABA). Three-week-old navel orange seedlings after grafting were treated by foliar sprays of $1 \mathrm{mM} \mathrm{SA}, 100 \mu \mathrm{M} \mathrm{JA}, 100 \mu \mathrm{M} \mathrm{ABA}$, or an equal volume of solvent [as a control (ck)]. The expression of CitYTH genes was analyzed by quantitative polymerase chain reaction after the seedlings were treated for 0,24 , and $48 \mathrm{~h}$. The $\mathrm{x}$ - and $\mathrm{y}$-axes represent the treat time (units $=$ hours) and relative expression (fold change relative to actin transcript values), respectively. The data presented are mean \pm SD of three independent experiments run in triplicate. The $*$ above the columns indicates a significant difference at the $P<0.05$ level.

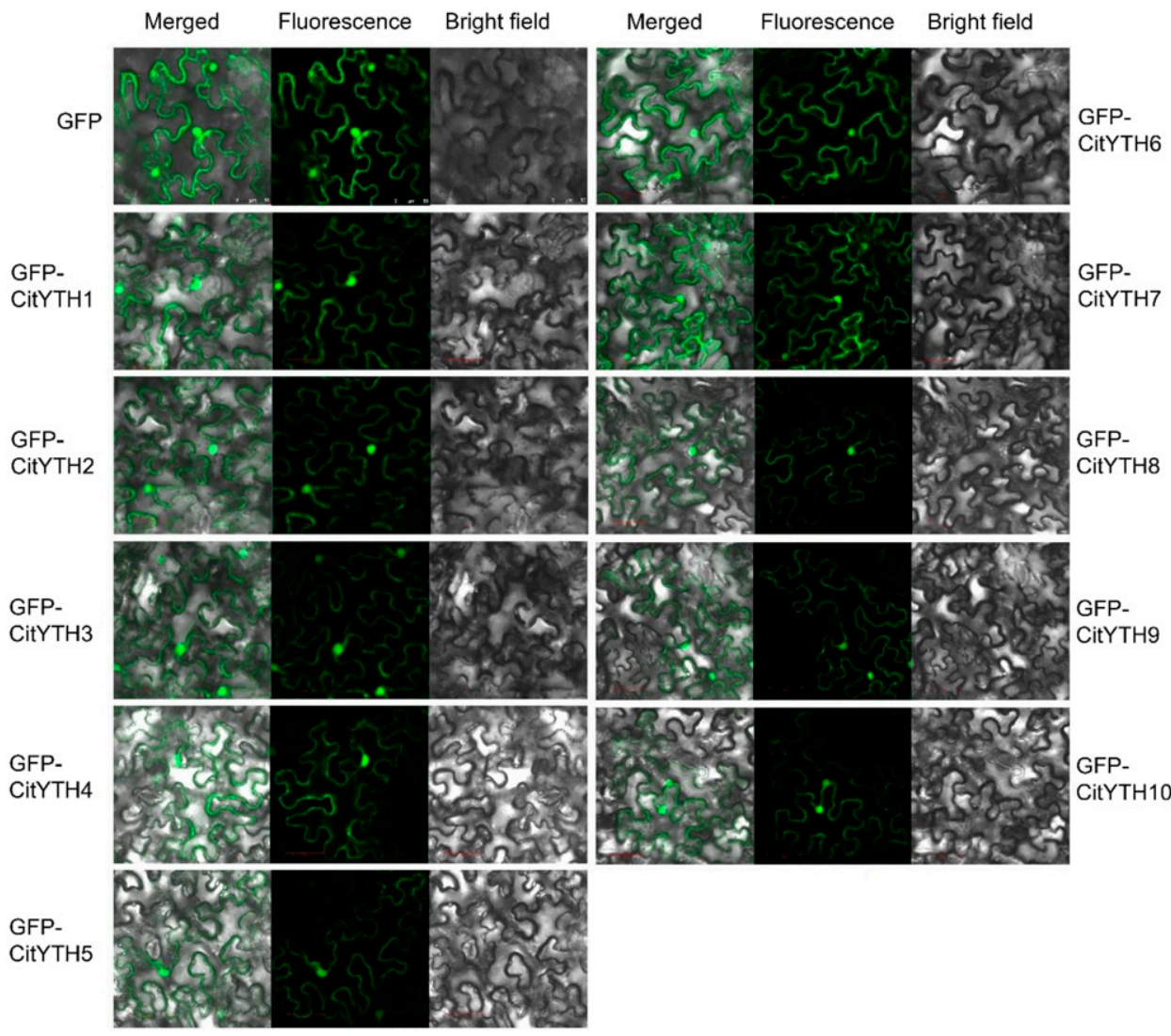

Fig. 9. Subcellular location of Citrus sinensis YTH domain-containing proteins. Nicotiana benthamiana leaves were transiently transformed with constructs harboring pFGC-Egfp:CitYTHs and pFGC-Egfp via agroinfiltration. Fluorescence of the target proteins was observed with confocal microscopy $36 \mathrm{~h}$ post infection.

protein domain, suggesting the YT521-B-like domains is a necessary and not just sufficient condition for judging YTH proteins.
A comprehensive gene expression analysis of YTH gene family members revealed that the YTH genes exhibited distinct expression patterns in various tissues of several plant species (Li et al., 2014). Some members of the YTH family exhibit organ-specific expression patterns or are not expressed in special tissues (e.g., CsYTH1 is not expressed in any tissues in cucumber) (Zhou et al., 2018). Furthermore, neither MdYTP9 nor MdYTP12 was detected in any of those tissues (Wang et al., 2014). In contrast, some YTH members, such as AtYTH genes, are highly expressed; however, there are exceptions; for example, the potential for AtYTH06 expression is relatively high in both the seeds, including in the embryo, endosperm, and testa and in the shoots (Li et al., 2014). The expression of $M d Y T H 2$ was highest in unfertilized ovaries and lowest in tendril bases. In addition, $M d Y T P 1$ transcripts were higher in the shoot apex and flowers than in other tissues (Wang et al., 2014). In the present study, 10 CitYTH genes were constitutively expressed in all tested tissues. For example, with the exception of those of CitYTH9, the expression levels of most of the CitYTH genes were lowest in the roots, whereas the expression levels of all 10 CitYTH genes were relatively high in the leaves; five CitYTH genes (Cit$Y T H 2,5,7,8,9$ ) were expressed in the flowers, and three CitYTH genes (CitYTH2, 5, 9) were expressed in the fruits (Fig. 4). YTH genes were expressed in all tested tissues and exhibited different expression patterns, suggesting that they may play different potential roles in different tissues.

The gene expression profiles at the different developmental stages or in response to different stresses may indicate gene function in relation to developmental and stress responses (Li et al., 2014). Previous studies have shown that the expression of YTH genes is highly developmentally regulated in plants. OsYTHs and AtYTHs exhibit high and low expression potential at the flowering stage and during different developmental stages (such as germinating seeds, seedlings, and rosettes) (Li et al., 2014). In apple, expression of MhYTP1 and MhYTP2 is induced during natural leaf senescence; 
compared with wild-type plants, the leaves of overexpression plants showed earlier yellowing and significantly lower chlorophyll concentrations in A. thaliana and apple. MhYTP2 can interact with acireductone dioxygenase 4 [an ethylene (ET) biosynthesis-related protein] to promote the progress of maturity (Wang et al., 2017a). The fruits of both MhYTPl and MhYTP2 transgenic tomato plants turned yellow earlier than did those of wild-type plants, indicating that these two YTH domain-containing genes can promote fruit ripening in tomato (Wang et al., 2017c). In two different cultivars, the expression of CitYTH5 and CitYTH10 was higher in peels of early cultivars, whereas the expression of CitYTH1, 7, 8, and 9 was lower (Fig. 5). Because the maturity period of the 'Gannan Zao' is shorter than that of the 'Newhall' by $\approx 1$ month, the results suggested that these affected genes may have a potential role played in peel and pulp development. However, the function of YTH genes in navel orange development and fruit ripening requires validation by further study.

In many species, YHT-containing genes can transcriptionally respond to abiotic stress, including high salinity, drought, heat, cold, and polyethylene glycol stress (Wang et al., 2014; Zhou et al., 2018). However, there is limited direct evidence showing that YTH genes play a role in different stress responses in plants. Overexpression of MhYTP1 or MhYTP2 makes plants more sensitive to $\mathrm{NaCl}$, but more resistant to nutrient deficits (Wang et al., 2017b). In addition, Wang et al. (2017c) reported that, compared with wild-type plants, plants overexpressing MhYTP1 or MhYTP2 were more sensitive to heat stress and high salinity but were more resistant to waterlogging, chilling, drought, and nutrient deficiency conditions. Moreover, both the MhYTP1 and MhYTP2 genes in those overexpression plants could be induced by various treatments (e.g., water logging, water deficit, and high salinity). In contrast, the abiotic stress also can affect the expression of YTHs. Several stress-related cis-elements, such as heat stress-responsive elements, droughtresponsive elements, defense- and stress-responsive elements (TC-rich repeats), anaerobic induction elements, and low temperature-responsive elements, all of which respond to external environmental stresses, have been found in the upstream regions of the promoters of YTH members ( $\mathrm{Li}$ et al., 2014; Wang et al., 2017a), and the MhYTP family was sensitive to chilling and $\mathrm{H}_{2} \mathrm{O}_{2}$ conditions and responsive to heat, water deficit, and salt treatment (Wang et al., 2014). Several AtYTH and OsYTH genes presented altered expression in response to abiotic stress. The expression profile of AtYTHO7 and AtYTH10 in the seedlings and flowers was dramatically induced at early stages during heat stress. In addition, increased expression levels of AtYTHO5 under cold stress, of AtYTHO2 under hypoxic stress, and of AtYTHO1 and AtYTHO2 under submergence stress also have been reported (Li et al., 2014). $\mathrm{ABA}$ is involved in the response to drought, salt, low temperature, and heat stress by inducing a series of signaling cascades. Studies suggest that osmotic stress imposed by high-salt or drought stress is transmitted through at least two pathways: ABA-dependent and ABA-independent pathways (Xiong and Zhu, 2002; Yamaguchi-Shinozaki and Shinozaki, 2006). ABA can also induce gene expression associated with stress responses (Busk and Pages, 1998). YTH members encompass a classic ABA-responsive element (ABRE, ACGTGT) found in the promoter region (Zhou et al., 2018). Thus, ABA could bind to the ABRE promoter to regulate gene expression. Several lines of evidence support the involvement of ABA in the YTH family in response to abiotic stresses. Expression of both MhYTP1 and MhYTP2 can be induced when ABA treatment is applied. Compared with wild-type plants, MhYTP1, 2 overexpression in plants was less sensitive to ABA treatment and has greater fresh weight and more roots. The expression patterns of AtYTHO6 in A. thaliana were also altered by treatment with cold, drought, heat, and osmotic stresses and by treatment with ABA (Li et al., 2014). In the present study, CitYTH2/4 was induced/suppressed by salt, drought, cold, and heat stresses; the expression of these genes was also induced by ABA, suggesting that CitYTH2/4 has various functions in response to multiple abiotic stresses via ABA signaling. However, serval CitYTH genes were affected by stress and not by ABA. This finding may suggest that CitYTH is also involved in ABA-independent pathways. Furthermore, we observed the induction or suppression of CitYTH family gene expression in response to the tested stress treatments, suggesting that crosstalk occurs between different stress signaling pathways.

SA and JA involved in defense against different biotic stressors have been well established (Atkinson and Urwin, 2012; Glazebrook, 2005; Laluk et al., 2012). Li et al. (2014) reported that treatment with ACC (a precursor of ET), ET, MeJA, or SA did not affect the expression patterns of all AtYTH genes in $A$. thaliana plants. However, in overexpressing plants MhYTH1 and MhYTH2, the expression of both of these genes was induced by treatment with SA and MeJA. Furthermore, transgenic plants showed more sensitivity to Diplocarpon mali infection, and expression of the JA signalingrelated genes PLD and COI1, the SA signaling-related genes PR1 and PR5, and the ET-responsive gene ERF3 in transgenic plants was altered, suggesting that $M h Y T H 1$ and $M h Y T H 2$ are involved in SA and MeJA/ET signaling (Wang et al., 2017a). The present results also showed that the expression of three CitYTH genes (CitYTH1, CitYTH2, and CitYTH10) was significantly altered in response to JA and SA. These findings were in accordance with that of $M h Y T H 1 / 2$, suggesting that these genes may be involved in JA and SA signaling. However, further studies are required to reveal the molecular mechanisms of CitYTHs in response to biotic stress by biochemical and genetic approaches.

RBPs coordinate the life of mRNAs, from birth in the nucleus to death in the cytoplasm, and play important roles in many regulatory aspects of cellular RNA metabolism (Wurth and Gebauer, 2014; Zhang et al., 2010), including RNA synthesis, folding/unfolding, modification, processing, and degradation. Thus, RBPs may function in both the nucleus and cytoplasm. MhYTP1 and MhYTP2 occur throughout the entire cell rather than within only specific compartments (Wang et al., 2017c). Otherwise, AtYTH5 and AtYTH7 in A. thaliana have also been shown to localize throughout the leaf cells of $N$. benthamiana (Li et al., 2014). Similarly, our subcellular results showed that the CitYTH proteins we selected were located in the nucleus and cytoplasm, which is consistent with their functional location.

In conclusion, 211 YTH genes were identified in 26 species that represent four major plant lineages in this study. Phylogenetic analysis revealed that these genes could be divided into eight subgroups. All of the YTH genes contain a YT521 domain and have different structures. Ten YTH genes were identified in $C$. sinensis. The expression profiles of these CitYTH genes were analyzed in different tissues and at different fruit developmental stages, and CitYTH genes 
displayed distinct expression patterns under heat, cold, salt, and drought stress. Furthermore, expression of the CitYTH genes in response to exogenous hormones was measured. Nuclear localization was also confirmed for five of these proteins encoded by these genes after transient expression in $N$. benthamiana cells.

\section{Literature Cited}

Alba, M. and M. Pagès. 1998. Plant proteins containing the RNArecognition motif. Trends Plant Sci. 3:15-21.

Ambrosone, A., A. Costa, A. Leone, and S. Grillo. 2012. Beyond transcription: RNA-binding proteins as emerging regulators of plant response to environmental constraints. Plant Sci. 182:12-18.

Atkinson, N.J. and P.E. Urwin. 2012. The interaction of plant biotic and abiotic stresses: From genes to the field. J. Expt. Bot. 63:35233543.

Braunschweig, U., S. Gueroussov, A.M. Plocik, B.R. Graveley, and B.J. Blencowe. 2013. Dynamic integration of splicing within gene regulatory pathways. Cell 152:1252-1269.

Busk, P.K. and M. Pages. 1998. Regulation of abscisic acid-induced transcription. Plant Mol. Biol. 37:425-435.

Calabretta, S. and S. Richard. 2015. Emerging roles of disordered sequences in RNA-binding proteins. Trends Biochem. Sci. 40:662-672. Cheong, C.G. and T.M.T. Hall. 2006. Engineering RNA sequence specificity of Pumilio repeats. Proc. Natl. Acad. Sci. USA 103:1363513639.

Cook, K.B., H. Kazan, K. Zuberi, Q. Morris, and T.R. Hughes. 2010. RBPDB: A database of RNA-binding specificities. Nucleic Acids Res. 39:301-308.

Duvick, J., A. Fu, U. Muppirala, M. Sabharwal, M.D. Wilkerson, C.J. Lawrence, C. Lushbough, and V. Brendel. 2008. PlantGDB: A resource for comparative plant genomics. Nucleic Acids Res. 36:D959-D965.

Enright, A.J. and C.A. Ouzounis. 2001. Functional associations of proteins in entire genomes by means of exhaustive detection of gene fusions. Genome Biol. 2:00341-00347.

Gasteiger, E., C. Hoogland, A. Gattiker, M.R. Wilkins, R.D. Appel, and A. Bairoch. 2005. Protein identification and analysis tools on the ExPASy server, p. 571-607. In: J.M. Walker (ed.). The proteomics protocols handbook. Humana Press, Totowa, NJ.

Glazebrook, J. 2005. Contrasting mechanisms of defense against biotrophic and necrotrophic pathogens. Annu. Rev. Phytopathol. 43:205-227.

Glisovic, T., J.L. Bachorik, J. Yong, and G. Dreyfuss. 2009. RNAbinding proteins and post-transcriptional gene regulation. FEBS Lett. 582:1977-1986.

Goodstein, D.M., S. Shu, R. Howson, R. Neupane, R.D. Hayes, J. Fazo, T. Mitros, W. Dirks, U. Hellsten, N. Putnam, and D.S. Rokhsar. 2011. Phytozome: A comparative platform for green plant genomics. Nucleic Acids Res. 40:D1178-D1186.

Hartmann, A.M., O. Nayler, F.W. Schwaiger, A. Obermeier, and S. Stamm. 1999. The interaction and colocalization of Sam68 with the splicing-associated factor YT521-B in nuclear dots is regulated by the Src family kinase p59fyn. Mol. Biol. Cell 10:3909-3926.

Herzel, L. and K.M. Neugebauer. 2015. Quantification of co-transcriptional splicing from RNA-Seq data. Methods 85:36-43.

Kim, Y.O., S. Pan, C.H. Jung, and H. Kang. 2007. A zinc fingercontaining glycine-rich RNA-binding protein, atRZ-1a, has a negative impact on seed germination and seedling growth of Arabidopsis thaliana under salt or drought stress conditions. Plant Cell Physiol. 48:1170-1181.

Laluk, K., K.V.S.K. Prasad, T. Savchenko, H. Celesnik, K. Dehesh, M. Levy, T. Mitchell-Olds, and A.S.N. Reddy. 2012. The calmodulinbinding transcription factor SIGNAL RESPONSIVE1 is a novel regulator of glucosinolate metabolism and herbivory tolerance in Arabidopsis. Plant Cell Physiol. 53:2008-2015.
Lamesch, P., T.Z. Berardini, D. Li, D. Swarbreck, C. Wilks, R. Sasidharan, R. Muller, K. Dreher, D.L. Alexander, M. GarciaHernandez, A.S. Karthikeyan, C.H. Lee, W.D. Nelson, L. Ploetz, S. Singh, A. Wensel, and E. Huala. 2012. The Arabidopsis Information Resource (TAIR): Improved gene annotation and new tools. Nucleic Acids Res. 40:D1202-D1210.

Larkin, M.A., G. Blackshields, N.P. Brown, R. Chenna, P.A. Mcgettigan, H. Mcwilliam, F. Valentin, I.M. Wallace, A. Wilm, R. Lopez, J.D. Thompson, T.J. Gibson, and D.G. Higgins. 2007. Clustal W and Clustal X version 2.0. Bioinformatics 23:2947-2948.

Li, D., H. Zhang, Y. Hong, L. Huang, X. Li, Y. Zhang, Z. Ouyang, and F. Song. 2014. Genome-wide identification, biochemical characterization, and expression analyses of the YTH domain-containing RNA-binding protein family in Arabidopsis and rice. Plant Mol. Biol. Rpt. 32:1169-1186.

Liu, Y., C.V. Hedvat, S. Mao, X.H. Zhu, J. Yao, H. Nguyen, A. Koff, and S.D. Nimer. 2006. The ETS protein MEF is regulated by phosphorylation-dependent proteolysis via the protein-ubiquitin ligase SCF ${ }^{\text {Skp2 }}$. Mol. Cell. Biol. 26:3114-3123.

Lorkovic, Z.J. 2009. Role of plant RNA-binding proteins in development, stress response and genome organization. Trends Plant Sci. 14:229-236.

Lorkovic, Z.J. and A. Barta. 2002. Genome analysis: RNA recognition motif (RRM) and $\mathrm{K}$ homology $(\mathrm{KH})$ domain RNA-binding proteins from the flowering plant Arabidopsis thaliana. Nucleic Acids Res. 30:623-635.

Lugassi, N., G. Kelly, L. Fidel, Y. Yaniv, Z. Attia, A. Levi, V. Alchanatis, M. Moshelion, E. Raveh, N. Carmi, and D. Granot. 2015. Expression of Arabidopsis hexokinase in citrus guard cells controls stomatal aperture and reduces transpiration. Front. Plant Sci. 6:1114. Marchler-Bauer, A., M.K. Derbyshire, N.R. Gonzales, S. Lu, F. Chitsaz, L.Y. Geer, R.C. Geer, J. He, M. Gwadz, D.I. Hurwitz, C.J. Lanczycki, F. Lu, G.H. Marchler, J.S. Song, N. Thanki, Z. Wang, R.A. Yamashita, D. Zhang, C. Zheng, and S.H. Bryant. 2014. CDD: NCBI's conserved domain database. Nucleic Acids Res. 43:D222-D226.

Marondedze, C., L. Thomas, N.L. Serrano, K.S. Lilley, and C. Gehring. 2016. The RNA-binding protein repertoire of Arabidopsis thaliana. Sci. Rpt. 6:29766.

Nicholas, K.B., H.B.J. Nicholas, and D.W.I. Deerfield. 1997. GeneDoc: Analysis and visualization of genetic variation. EMBnet News 4:14.

Owttrim, W.G. 2006. RNA helicases and abiotic stress. Nucleic Acids Res. 34:3220-3230.

Pascual, M.B., J. El-Azaz, F.N. de la Torre, R.A. Cañas, C. Avila, and F.M. Cánovas. 2016. Biosynthesis and metabolic fate of phenylalanine in conifers. Front. Plant Sci. 7:1030.

Qu, J., S.G. Kang, W. Wang, K. Musier-Forsyth, and J.C. Jang. 2014. The Arabidopsis thaliana tandem zinc finger 1 (AtTZF1) protein in RNA binding and decay. Plant J. 78:452-467.

Shahbaz, M. and M. Ashraf. 2013. Improving salinity tolerance in cereals. Crit. Rev. Plant Sci. 32:237-249.

Sharan, M., K.U. Förstner, A. Eulalio, and J. Vogel. 2017. APRICOT: An integrated computational pipeline for the sequence-based identification and characterization of RNA-binding proteins. Nucleic Acids Res. 45:e96.

Shi, H., X. Wang, Z. Lu, B.S. Zhao, H. Ma, P.J. Hsu, C. Liu, and C. He. 2017. YTHDF3 facilitates translation and decay of N 6-methyladenosinemodified RNA. Cell Res. 27:315-328.

Shi, Y. and J.L. Manley. 2015. The end of the message: Multiple protein-RNA interactions define the mRNA polyadenylation site. Genes Dev. 29:889-897.

Song, J.J., J. Liu, N.H. Tolia, J. Schneiderman, S.K. Smith, R.A. Martienssen, G.J. Hannon, and L. Joshua-Tor. 2003. The crystal structure of the Argonaute2 PAZ domain reveals an RNA binding motif in RNAi effector complexes. Nat. Struct. Biol. 10:1026-1032. Stoilov, P., I. Rafalska, and S. Stamm. 2002. YTH: A new domain in nuclear proteins. Trends Biochem. Sci. 27:495-497.

Tam, P.P., I.H. Barrette-Ng, D.M. Simon, M.W. Tam, A.L. Ang, and D.G. Muench. 2010. The Puf family of RNA-binding proteins in 
plants: Phylogeny, structural modeling, activity and subcellular localization. BMC Plant Biol. 10:44.

Tamura, K., G. Stecher, D. Peterson, A. Filipski, and S. Kumar. 2013. MEGA6: Molecular evolutionary genetics analysis version 6.0. Mol. Biol. Evol. 30:2725-2729.

Wang, N., T. Guo, X. Sun, X. Jia, P. Wang, Y. Shao, B. Liang, and F. Ma. 2017a. Functions of two Malus hupehensis (Pamp.) Rehd. YTPs (MhYTP1 and MhYTP2) in biotic- and abiotic-stress responses. Plant Sci. 261:18-27.

Wang, N., T. Guo, P. Wang, X. Sun, Y. Shao, X. Jia, B. Liang, X. Gong, and F. Ma. 2017b. MhYTP1 and MhYTP2 from apple confer tolerance to multiple abiotic stresses in Arabidopsis thaliana. Front. Plant Sci. 8:1367.

Wang, N., T. Guo, P. Wang, X. Sun, Y. Shao, B. Liang, X. Jia, X. Gong, and F. Ma. 2017c. Functional analysis of apple MhYTP1 and MhYTP2 genes in leaf senescence and fruit ripening. Scientia Hort. 221:23-32. Wang, N., Z. Yue, D. Liang, and F. Ma. 2014. Genome-wide identification of members in the YTH domain-containing RNAbinding protein family in apple and expression analysis of their responsiveness to senescence and abiotic stresses. Gene 538:292-305.

Wang, Y., Z. Wang, and T.M.T. Hall. 2013. Engineered proteins with Pumilio/fem-3 mRNA binding factor scaffold to manipulate RNA metabolism. FEBS J. 280:3755-3767.

Warnes, G.R., B. Bolker, L. Bonebakker, R. Gentleman, W.H.A. Liaw, T. Lumley, M. Maechler, A. Magnusson, S. Moeller, M. Schwartz, and B. Venables. 2013. Gplots: Various R programming tools for plotting data, v2.12.1. 22 Oct. 2018. <http://cran.r-project.org/web/ packages/gplots/index.html>.

Wigington, C.P., J. Jung, E.A. Rye, S.L. Belauret, A.M. Philpot, Y. Feng, P.J. Santangelo, and A.H. Corbett. 2014. Post-transcriptional regulation of programmed cell death 4 (PDCD4) mRNA by the RNA binding proteins human antigen $\mathrm{R}(\mathrm{HuR})$ and $\mathrm{T}$-cell intracellular antigen 1 (TIA1). J. Biol. Chem. 290:3468-3487.

Wurth, L. and F. Gebauer. 2014. RNA-binding proteins, multifaceted translational regulators in cancer. Biochim. Biophys. Acta 1849:881-886.

Xiong, L. and J.K. Zhu. 2002. Molecular and genetic aspects of plant responses to osmotic stress. Plant Cell Environ. 25:131-139.

Yamaguchi-Shinozaki, K. and K. Shinozaki. 2006. Transcriptional regulatory networks in cellular responses and tolerance to dehydration and cold stresses. Annu. Rev. Plant Biol. 57:781-803.

Zhang, Z., D. Theler, K.H. Kaminska, M. Hiller, P. de la Grange, R. Pudimat, I. Rafalska, B. Heinrich, J.M. Bujnicki, F.H.T. Allain, and S. Stamm. 2010. The YTH domain is a novel RNA binding domain. J. Biol. Chem. 285:14701-14710.

Zhou, Y., L. Hu, L. Jiang, and S. Liu. 2018. Genome-wide identification and expression analysis of YTH domain-containing RNAbinding protein family in cucumber (Cucumis sativus). Genes Genomics 40:579-589.

Zhu, J.K. 2016. Abiotic stress signaling and responses in plants. Cell 167:313-324. 
Supplemental Table 1. Primers used for quantitative polymerase chain reaction (qPCR) analysis of Citrus sinensis YTH genes expression and subcellular localization. For qPCR, CitActin was used as reference gene (Lugassi et al., 2015).

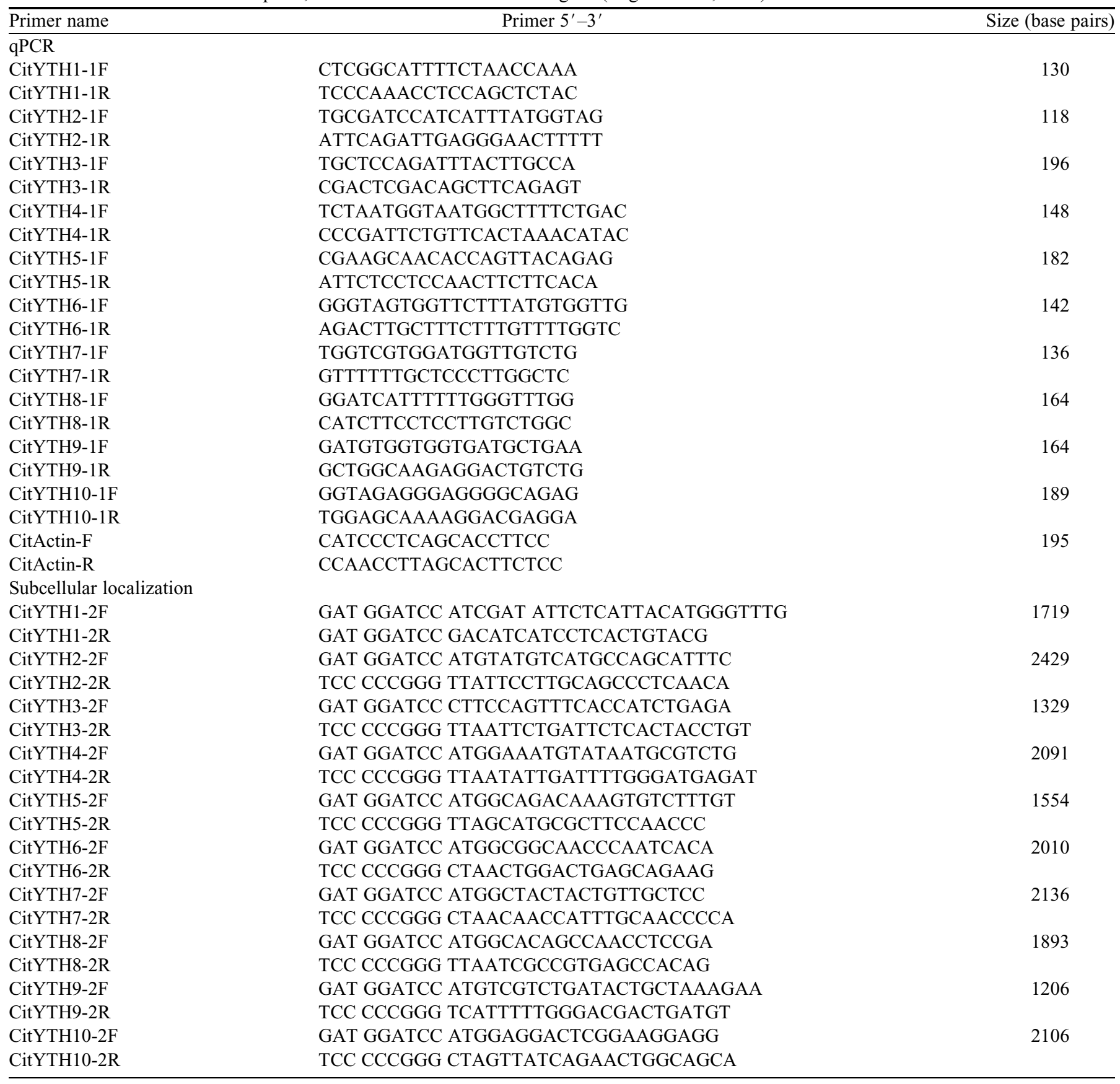


Supplemental Table 2. Information of YTH domain-containing proteins identified in in this study. Protein sequences of YTH domain-containing proteins were obtained from the Phytozome database (Goodstein et al., 2011).

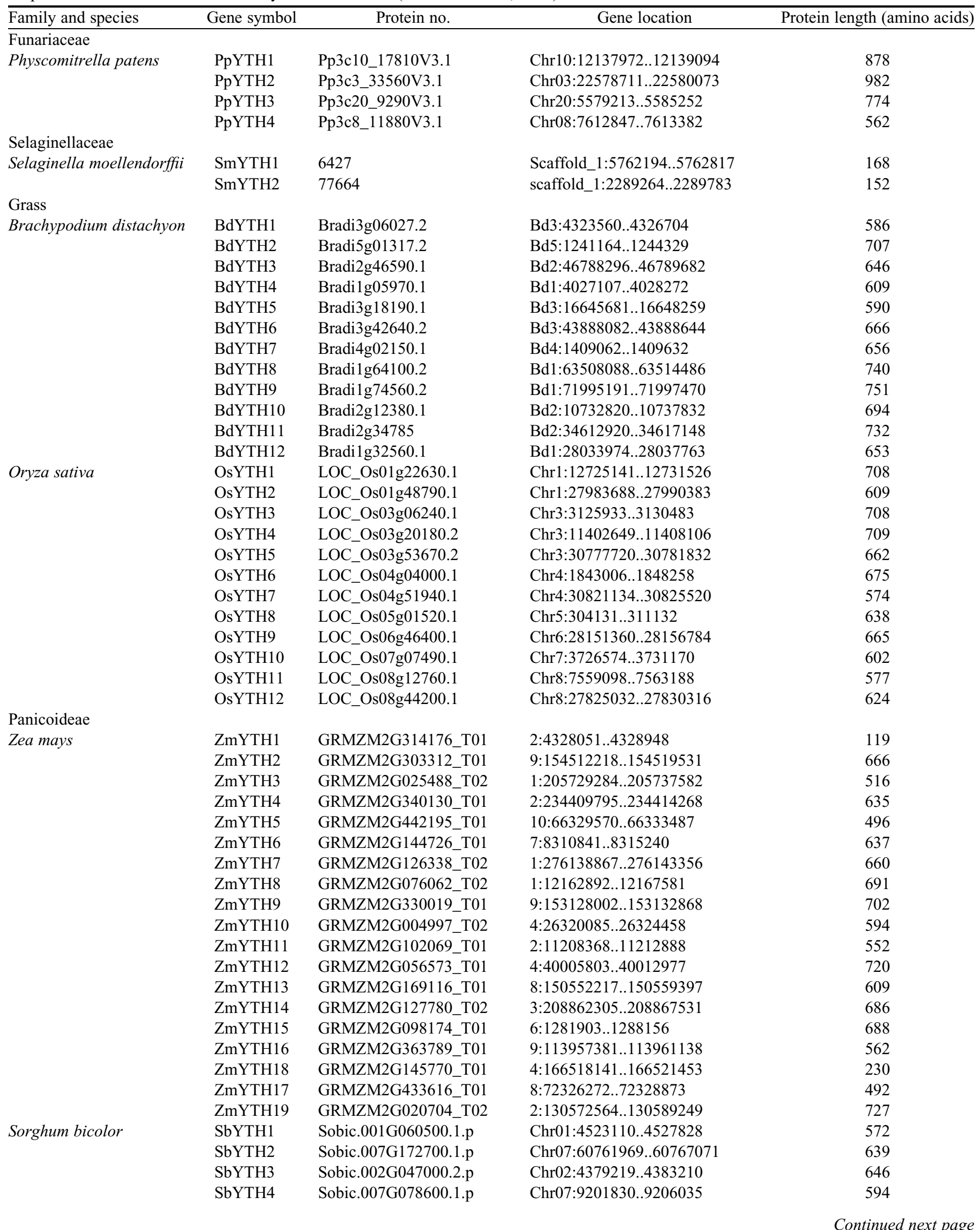


Supplemental Table 2. Continued.

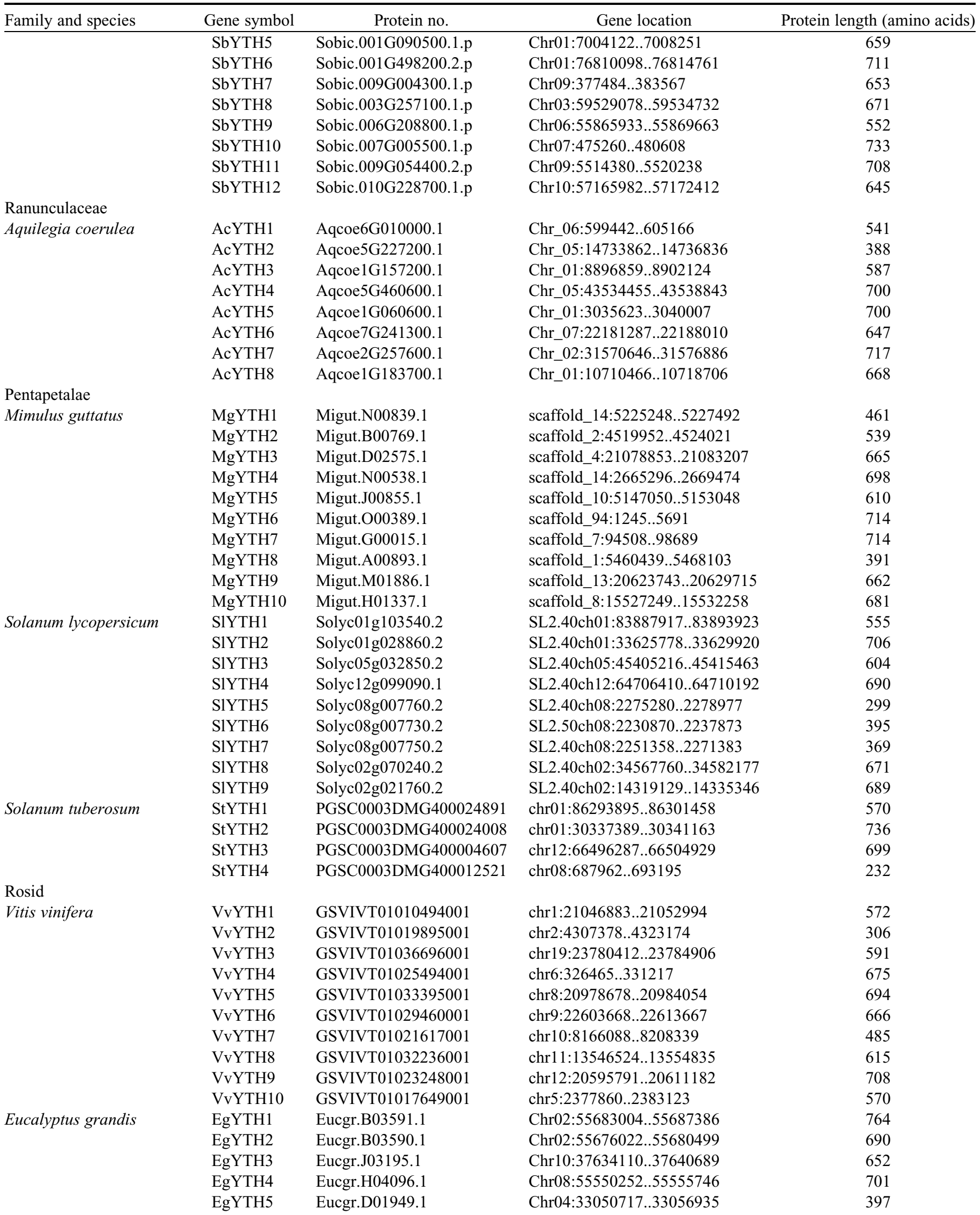

Continued next page 
Supplemental Table 2. Continued.

\begin{tabular}{|c|c|c|c|c|}
\hline Family and species & Gene symbol & Protein no. & Gene location & Protein length (amino acids) \\
\hline & EgYTH6 & Eucgr.E02496.1 & Chr05:32701248..32710600 & 723 \\
\hline \multicolumn{5}{|l|}{ Rutaceae } \\
\hline \multirow[t]{8}{*}{ Citrus sinensis } & CitYTH1 & orange1.1g008255m & $7,160,400 . .7,170,600$ & 701 \\
\hline & CitYTH3 & orange $1.1 \mathrm{~g} 038459 \mathrm{~m}$ & $2,895,200 . .2,908,800$ & 442 \\
\hline & CitYTH4 & orange $1.1 \mathrm{~g} 005441 \mathrm{~m}$ & $1,636,500 . .1,647,700$ & 711 \\
\hline & CitYTH5 & orange1.1g010141m & $29,401,300 . .29,414,400$ & 517 \\
\hline & CitYTH7 & orange1.1g005158m & $352,700 . .364,000$ & 696 \\
\hline & CitYTH8 & orange1.1g006798m & $7,023,100 . .7,040,400$ & 630 \\
\hline & CitYTH9 & orange $1.1 \mathrm{~g} 015749 \mathrm{~m}$ & $6,833,100 . .6,847,700$ & 401 \\
\hline & CitYTH10 & orange1.1g005338m & $106,600 . .118,100$ & 472 \\
\hline \multirow[t]{6}{*}{ Citrus clementina } & CcYTH1 & Ciclev10000713m & scaffold_5:5309895..5315706 & 572 \\
\hline & $\mathrm{CcYTH} 2$ & Ciclev10004597m & scaffold_9:31193246..31199360 & 594 \\
\hline & CcYTH7 & Ciclev10019348m & scaffold_3:41345629..41350936 & 611 \\
\hline & CcYTH8 & Ciclev10030951m & scaffold_4:21192983..21199939 & 630 \\
\hline & CcYTH9 & Ciclev $10020457 \mathrm{~m}$ & scaffold_3:6546647..6552193 & 401 \\
\hline & CcYTH10 & Ciclev10014454m & scaffold_2:31489811..31497594 & 701 \\
\hline \multicolumn{5}{|l|}{ Brassicales-Malvales } \\
\hline \multirow[t]{6}{*}{ Theobroma cacao } & TcYTH1 & Thecc1EG006167t1 & scaffold_2:1059280..1066411 & 573 \\
\hline & TcYTH2 & Thecc1EG039865t1 & scaffold_9:24125408..24130041 & 742 \\
\hline & TcYTH3 & Thecc1EG026461t1 & scaffold_5:38374403..38378754 & 622 \\
\hline & TcYTH4 & Thecc1EG027659t6 & scaffold_6:5912196..5918919 & 652 \\
\hline & TcYTH5 & Thecc1EG021457t1 & scaffold_4:33165146..33169610 & 659 \\
\hline & TcYTH6 & Thecc1EG008783t1 & scaffold_2:16988579..16996466 & 634 \\
\hline & GrYTH5 & Gorai.009G120700.1 & Chr09:8935247..8942392 & 654 \\
\hline & GrYTH6 & Gorai.011G001700.1 & Chr11:161861..168102 & 648 \\
\hline & GrYTH7 & Gorai.007G289200.1 & Chr07:49545172..49551745 & 622 \\
\hline & GrYTH8 & Gorai.013G123200.1 & Chr13:31722245..31726598 & 606 \\
\hline & GrYTH9 & Gorai.005G070500.1 & Chr05:7641980..7646032 & 654 \\
\hline & GrYTH10 & Gorai.002G031800.1 & Chr02:2370851..2374924 & 654 \\
\hline & GrYTH11 & Gorai.002G182900.1 & Chr02:48153196..48160671 & 625 \\
\hline & GrYTH12 & Gorai.004G205800.1 & Chr04:53583839..53588598 & 386 \\
\hline & GrYTH13 & Gorai.008G046800.1 & Chr08:6332699..6338419 & 701 \\
\hline \multicolumn{5}{|l|}{ Brassicaceae } \\
\hline \multirow[t]{11}{*}{ Brassica rapa } & BrYTH1 & Brara.F00638.1 & A06:3655226..3658829 & 502 \\
\hline & BrYTH2 & Brara.G02587.1 & A07:21382343..21384514 & 421 \\
\hline & BrYTH3 & Brara.A03796.1 & A01:30159742..30162154 & 406 \\
\hline & BrYTH4 & Brara.G03650.1 & A07:27015669..27018099 & 443 \\
\hline & BrYTH5 & Brara.E03479.1 & A05:27549589..27551472 & 397 \\
\hline & BrYTH6 & Brara.B02362.1 & A02:15455762..15458405 & 448 \\
\hline & BrYTH7 & Brara.B02570.1 & A02:18182697..18184972 & 447 \\
\hline & BrYTH8 & Brara.G02088.1 & A07:18521873..18524730 & 494 \\
\hline & BrYTH9 & Brara.E02771.1 & A05:23983684..23987129 & 644 \\
\hline & BrYTH10 & Brara.C03469.1 & A03:17568871..17572245 & 639 \\
\hline & BrYTH11 & Brara.A03258.1 & A01:27170859..27174022 & 655 \\
\hline
\end{tabular}

Continued next page 
Supplemental Table 2. Continued.

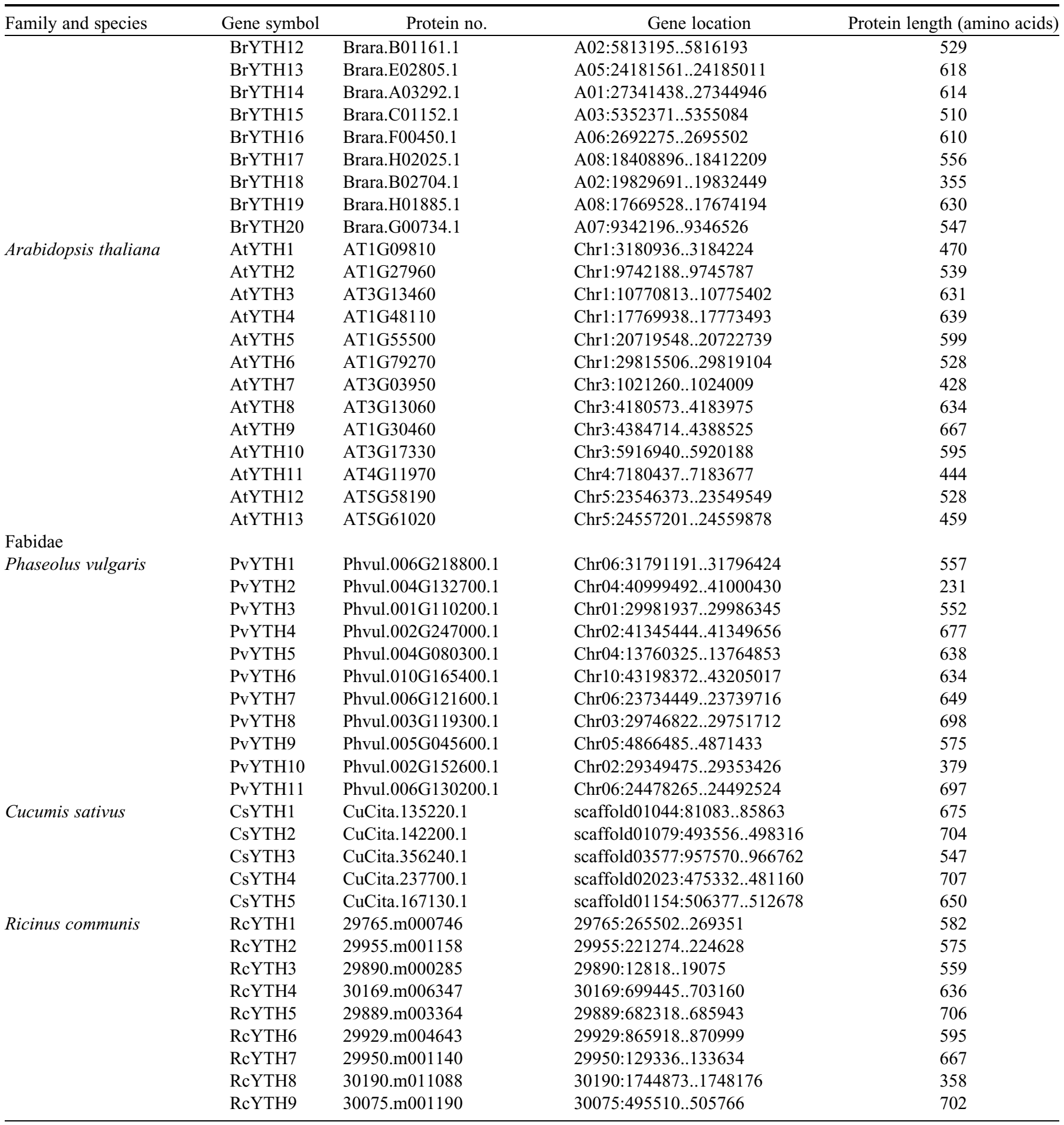

